University of Nebraska - Lincoln

DigitalCommons@University of Nebraska - Lincoln

2010

\title{
Multiscale modeling of the impact of textile fabrics based on hybrid element analysis
}

\author{
Gaurav Nilakantan \\ University of Delaware \\ Michael Keefe \\ University of Delaware \\ Travis A. Bogetti \\ US Army Research Laboratory \\ John W. Gillespie Jr. \\ University of Delaware, gillespi@udel.edu
}

Follow this and additional works at: https://digitalcommons.unl.edu/usarmyresearch

Part of the Operations Research, Systems Engineering and Industrial Engineering Commons

Nilakantan, Gaurav; Keefe, Michael; Bogetti, Travis A.; and Gillespie, John W. Jr., "Multiscale modeling of the impact of textile fabrics based on hybrid element analysis" (2010). US Army Research. 141.

https://digitalcommons.unl.edu/usarmyresearch/141

This Article is brought to you for free and open access by the U.S. Department of Defense at DigitalCommons@University of Nebraska - Lincoln. It has been accepted for inclusion in US Army Research by an authorized administrator of DigitalCommons@University of Nebraska - Lincoln. 


\title{
Multiscale modeling of the impact of textile fabrics based on hybrid element analysis
}

\author{
Gaurav Nilakantan ${ }^{\mathrm{a}, \mathrm{b}}$, Michael Keefe ${ }^{\mathrm{a}, \mathrm{d}}$, Travis A. Bogetti ${ }^{\mathrm{e}}$, John W. Gillespie Jr. ${ }^{\mathrm{a}, \mathrm{b}, \mathrm{c}, *}$ \\ ${ }^{a}$ Center for Composite Materials, University of Delaware, Newark, DE 19716, USA \\ ${ }^{\mathrm{b}}$ Department of Materials Science and Engineering, University of Delaware, Newark, DE 19716, USA \\ ${ }^{\mathrm{C}}$ Department of Civil and Environmental Engineering, University of Delaware, Newark, DE 19716, USA \\ d Department of Mechanical Engineering, University of Delaware, Newark, DE 19716, USA \\ ${ }^{\mathrm{e}}$ US Army Research Laboratory, Aberdeen Proving Ground, MD 21005, USA
}

\section{A R T I C L E I N F O}

\section{Article history:}

Received 28 November 2008

Received in revised form

28 February 2010

Accepted 20 April 2010

Available online 19 May 2010

\section{Keywords:}

Textile composite

Woven fabric

Finite element analysis (FEA)

Impact

Multi scale modeling

\begin{abstract}
A B S T R A C T
In this work, a multi scale modeling approach has been developed to simulate the impact of woven fabrics using a finite element (FE) analysis. A yarn level of resolution is used in the model. This approach, referred to as the hybrid element analysis (HEA) is based on decreasing the complexity of the finite element model with distance away from the impact zone based on the multiscale nature of the fabric architecture and the physics of the impact event. Solid elements are used to discretize the yarns around the impact region, which transition to shell elements in the surrounding region. A new method for modeling the shell yarns is incorporated that more accurately represents the contours of the yarn cross section. Impedances have been matched across the solid-shell interface to prevent interfacial reflections of the longitudinal strain wave. The HEA method is validated by first applying it to the FE model of a single yarn for which an analytical solution is known. The HEA method is then applied to a woven fabric model and validated by comparing it against a baseline model consisting of yarns discretized using only solid elements.
\end{abstract}

(c) 2010 Elsevier Ltd. All rights reserved.

\section{Introduction}

Various applications have utilized the effective penetration resistance and impact energy dissipation offered by woven fabrics comprised of high performance materials such as Kevlar, Zylon, and Twaron. These applications include protective clothing for personnel extremity protection and materials for turbine fragment containment. These high performance materials have a high strength to weight ratio, high stiffness, and high strength, making them ideal for these applications. Considerable research has been conducted to understand the intricate architecture of these fabrics, as well as for capturing their non-linear behavior and complex interactions during in-plane loading and transverse impact. Various analytical, numerical and experimental approaches have been employed. Some of these approaches and the factors that affect the impact response of flexible woven composites have been outlined

\footnotetext{
* Corresponding author. Center for Composite Materials, University of Delaware, Newark, DE 19716, USA. Tel.: +1 302831 8702; fax: +1 3028318525.
}

E-mail address: gillespi@udel.edu (J.W. Gillespie Jr.). in the reviews by Tabiei and Nilakantan [1], and by Cheeseman and Bogetti [2].

There are several techniques used to represent fabric behavior. Single scale modeling techniques include representing the entire fabric as homogenized membranes [3-5], explicitly capturing yarn level architecture [6-8], and capturing filament level architecture $[9,10]$. Neglecting yarn architecture, as is the case when representing the fabric by a homogenous membrane leads to excellent computational efficiency, but these approaches often use oversimplifying assumptions. For example individual yarn failure, capturing phenomena such as yarn pullout and sliding, accounting for transverse yarn compression and thickness changes, and computing the energy dissipated by frictional sliding between yarns are not possible with these models. Frictional effects have been shown to have an important effect on the fabric response to impact and researchers have been investigating methods to alter inter yarn frictional properties with a view to improving the energy dissipation capabilities of these fabrics [11,12]. On the other hand, models that explicitly capture yarn architecture are more accurate and can account for individual yarn motion and interactions. However these models usually require enormous computational resources in order to be able to model multi layer fabric systems of 
realistic dimensions. Such computational resources are currently unavailable. Some finite element (FE) codes also tend to become unstable when dealing with the enormous number of degrees of freedom associated with such models.

More recently, multi scale modeling techniques have emerged that attempt to balance the inequity between accuracy and fineness of modeling resolution with computational expense. Barauskas and Abraitiene $[13,14]$ used a mezzo- and macro-mechanical model that used thin shell elements to model yarns at the impact point and roughly meshed uniform orthotropic thin shells for zones far away from the impact point. A tied interface was used to couple both regions. However their model could not adequately capture changes in the yarn thickness and cross sectional shape, and their model required a manipulation of the contact algorithm's penalty coefficient in an attempt to mimic the through thickness deformation of the yarns. The selection of the material model and corresponding material parameters, especially in the zones remote from the impact site is in an arbitrary manner and this is reflected by large errors in the wave propagation speeds which directly affect the accuracy of results, especially in high rate deformation conditions. No comparison has been made against a baseline numerical model in terms of either simulation results such as projectile velocity history and energy interaction histories or simulation run times, although the purpose of such a model is to reflect savings in computational expense while maintaining accuracy. It is essential that a FE model be predictive in nature and not dependent on arbitrary adjustment of input parameters to fit experimental or baseline data. Rao et al. [15] present a multi scale model that uses a 'center-cross' modeling approach. Yarns are discretely modeled using solid elements within a central cross or local region which extends up to the fabric boundaries, while a homogenized layer modeled with two layers of solid elements is used in the global region, which is the region outside the central cross shape. This allows for some degree of yarn sliding and yarn pullout, especially in cases where the fabric is gripped on two ends. However maintaining local yarn architecture modeled using solid elements all the way to the fabric boundaries may be impractical in terms of computational expense. Their model uses multiple layers of solid elements in the global region leading to a far more computationally expensive model than if shell elements were used in the global region. Their multi scale model compares well to their baseline numerical model, which is comprised wholly of yarns modeled using solid elements.

As such the field of multi scale numerical modeling of the impact of flexible woven fabrics is fairly recent, and much research still needs to be conducted in order to improve accuracy and establish a consistent modeling methodology, while reducing computational expense. To this end, a multi scale numerical model that uses a new technique entitled 'hybrid element analysis' is developed. The HEA method is compared to a baseline numerical model where a fabric has been completely modeled with a yarn level resolution using solid elements. The purpose of the HEA model is to accurately reproduce the results of the baseline numerical model, but at a much lower computational expense. This study introduces the HEA approach starting with a single yarn model and extending it to a fabric model with a yarn level architecture used throughout. This is referred to as the 'single scale HEA'.

\section{Modeling of yarns using shell elements}

Solid or hexahedral finite elements are a common choice when modeling yarns. Fig. 1 displays the FE mesh of an uncrimped woven yarn from a plain weave fabric modeled using solid finite elements. A mesh sensitivity study was conducted to decide a suitable mesh scheme that balances computational expense with accuracy of

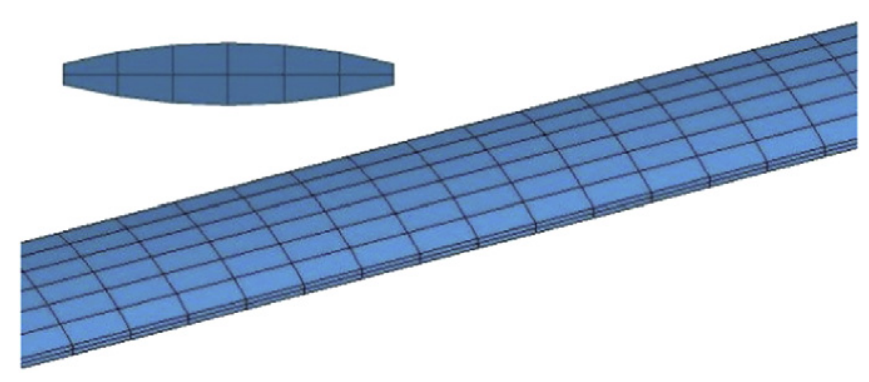

Fig. 1. FE mesh of a yarn modeled using solid finite elements.

results. Six elements are used across the yarn width to sufficiently capture the curved shape of the yarn cross section, and two elements are used through the thickness. Since yarns are essentially very thin structures, in order to maintain good element aspect ratios a large number of elements need to be used along the yarn length so that the element dimension along the length is the same order of magnitude as the element dimension across the width. This is also important in order to maintain smooth contacting surfaces between two orthogonal yarns that slide past one another. This makes using only solid elements a computationally intensive choice when modeling yarns of very large lineal dimensions.

The principal idea behind using a shell element is to collapse a three dimensional object into two dimensions, which also enables the modeling of very thin structures. Shell elements are computationally less intensive than solid elements, thereby making them useful for modeling yarns. Shell elements have a minimum of two integration points through the thickness allowing them to account for both bending stiffness and thickness changes unlike membrane elements that have only one through thickness integration point. The thickness change in a shell element is determined from the computation of the through thickness strain, which in turn is computed from the in-plane normal stresses and Poisson ratios. Thus the transverse compression of a shell element is not updated separately since the plane stress condition mandates that the through thickness stress is zero, rather it depends on the membrane straining. However the observed thickness changes in yarns are mainly due to transverse compressions as opposed to longitudinal straining. Thus the constant thickness of a shell element is a potential limitation when trying to use shell elements in regions where capturing transverse yarn compressions is important, such as directly under the projectile at the impact location. However shell elements are easily able to capture the axial tensile deformations of a yarn modeled with solid elements.

Previous approaches [13,16] to modeling yarns using shell elements are shown in Fig. 2a. As can be observed, the cross sectional shape is not realistically modeled. Each shell element and its corresponding nodes have been assigned a uniform thickness t-i to t-iii. There are thickness jumps at the shell element boundaries [16]. This is not desirable in the impact of textile fabric structures where it is essential to accurately capture inter yarn sliding interactions which require realistic representations of the yarn cross section. Another drawback is the loss of yarn material at the regions of the thickness jumps leading to a loss in yarn mass. Two possibilities of conserving the yarn mass are to either artificially increase the yarn material density or to increase the thickness of the shell elements. The former leads to a different longitudinal strain wave velocity and prediction of longitudinal stresses within the yarn, while the latter leads to inter yarn penetrations at the yarn crossover locations in a fabric, both of which are undesirable. A new modeling approach is illustrated in Fig. $2 b$, where a yarn has been modeled using the same number of shell elements across the width, with non-uniform nodal thicknesses $\mathrm{t} 1-\mathrm{t} 4$. These non- 
a

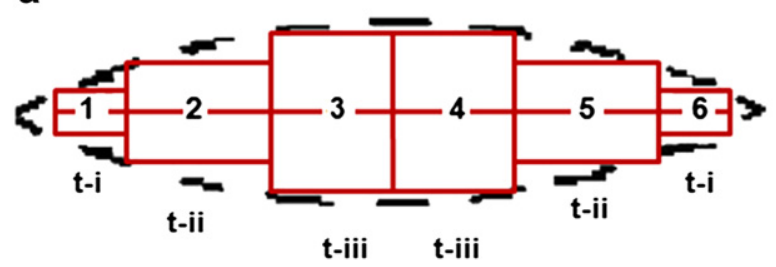

b

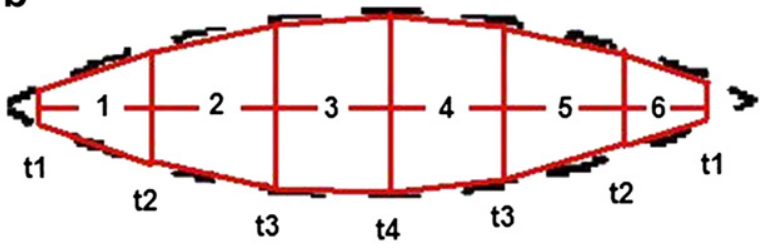

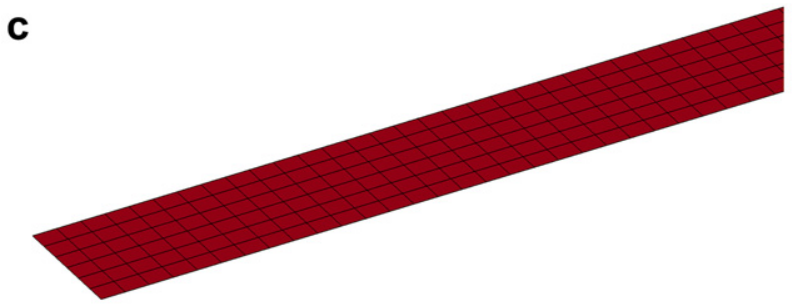

Fig. 2. Yarn cross section using shell elements with (a) uniform nodal thicknesses, (b) non-uniform nodal thicknesses. (c) FE mesh of a yarn modeled using shell elements.

uniform nodal thicknesses have been specified for each shell element, so that it closely approximates the cross section of a yarn. Such a modeling approach leads to a more accurate representation of the yarn geometry and mass. The preprocessor DYNAYarn [17] was used to create the FE mesh and automatically assign the nonuniform nodal thicknesses to each shell element. Fig. 2c displays the FE mesh of a yarn modeled using shell elements which is equivalent to the yarn modeled using solid elements in Fig. 1.

To compare this new method of modeling yarns with shell elements (see Fig. 2b) with the previous method (see Fig. 2a), a high rate yarn tensile test is simulated using the dynamic finite element code LS-DYNA. Consider the case of a $50 \mathrm{~mm}$ long uncrimped yarn gripped at one end and pulled at the other end in the $x$-direction with a constant velocity of $20 \mathrm{~m} / \mathrm{s}$. The simulation is run for $50 \mu \mathrm{s}$ which results in a net displacement of $1 \mathrm{~mm}$ at the pulled end. A linearly elastic orthotropic material model (Mat \#2 in LS-DYNA) is assigned to the yarn with the following properties: longitudinal elastic modulus $\left(E_{x}\right)$ of $89 \mathrm{GPa}$, density $(\rho)$ of $1440 \mathrm{~kg} / \mathrm{m}^{3}$, and strain to failure $\left(\varepsilon_{\mathrm{f}}\right)$ of $3.1 \%$. According to Ref. [18], an orthotropic elastic continuum can be used to model a continuous filament yarn provided the transverse moduli $\left(E_{y}, E_{z}\right)$ are very small compared to the longitudinal elastic modulus $\left(E_{x}\right)$, and with zero Poisson ratios and shear moduli $\left(G_{x y}, G_{y z}, G_{z x}\right)$. This approach essentially implies a one dimensional response similar to a cable or beam wherein the longitudinal response is the most predominant. However in a finite element analysis, assigning the shear moduli to zero could lead to element hourglassing or zero strain energy modes and so a small value usually needs to be assigned to the material. For lack of better data, shear moduli $\left(G_{x y}, G_{y z}, G_{z x}\right)$ of $3280 \mathrm{MPa}$, and zero Poisson ratios have been selected [19]. Here the $x$-axis is along the length direction, the $y$-axis is along the thickness direction, while the $z$ axis is along the width direction. Four test cases are studied where the yarn is modeled with (\#1) solid elements which serve as the baseline, (\#2) our new method with shell elements which is also part of the HEA approach, (\#3) previous method with shell elements, see Fig. 2a, and (\#4) previous method with shell elements where the density has been increased to account for the lost yarn mass due to the thickness jumps. Fig. 3 compares the total yarn mass in all four cases. The yarn masses have been normalized with respect to case \#1. As can be seen, there is about a $12 \%$ loss in mass due to the thickness jumps in case \#3 which is rectified in case \#4 by increasing the yarn density from $1440.00 \mathrm{~kg} / \mathrm{m}^{3}$ to $1636.58 \mathrm{~kg} / \mathrm{m}^{3}$. Table 1 displays the yarn thickness at various locations across the yarn width for each case, with reference to Figs. $1,2 \mathrm{a}$ and $\mathrm{b}$ respectively. Before we compare the results between the four cases, we will first review some 1-d wave propagation theory to formulate some analytical predictions for comparison with the numerical predictions, with respect to the case of a yarn held at one end and pulled at a constant velocity at the other end.

Wave propagation in bars is well approximated by the one dimensional wave equation [20]

$\frac{\partial^{2} u}{\partial t^{2}}=c^{2} \frac{\partial^{2} u}{\partial x^{2}}$

which can be factored into a first order equation for this case study as

$\frac{\partial u}{\partial t}=c \frac{\partial u}{\partial x}$

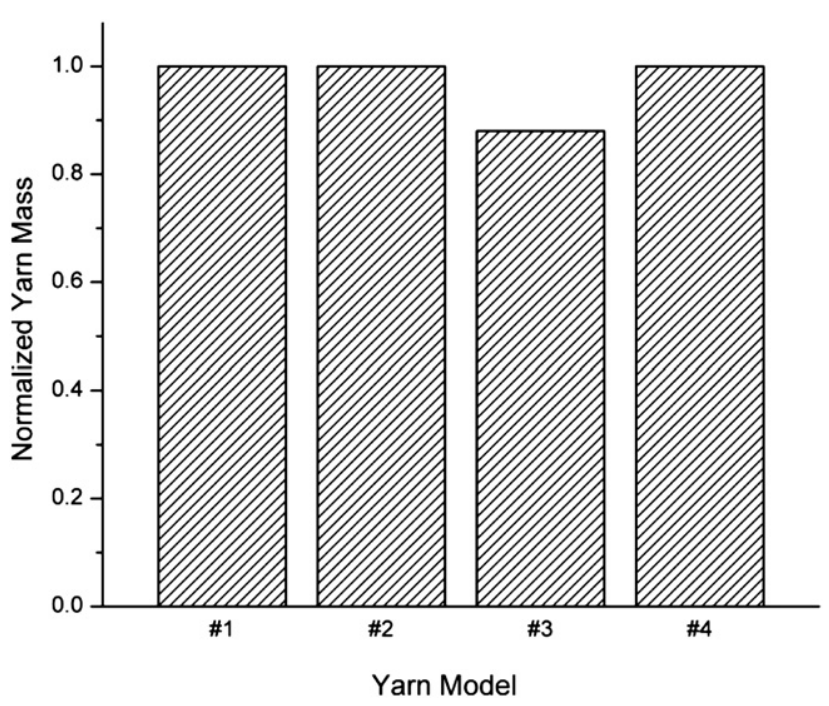

Fig. 3. Comparison of yarn mass. 
Table 1

Thicknesses across the yarn width.

\begin{tabular}{llllll}
\hline \multicolumn{2}{l}{ Baseline (solids) } & \multicolumn{2}{l}{$\begin{array}{l}\text { Previous method } \\
\text { (shells) }\end{array}$} & \multicolumn{2}{l}{ New method (shells) } \\
\hline Node & $t(\mathrm{~mm})$ & Element & $t(\mathrm{~mm})$ & Node & $t(\mathrm{~mm})$ \\
\hline $\mathrm{t} 1$ & 0.049366 & $\mathrm{t}-\mathrm{i}$ & 0.049366 & $\mathrm{t} 1$ & 0.049366 \\
$\mathrm{t} 2$ & 0.084034 & $\mathrm{t}-\mathrm{ii}$ & 0.084034 & $\mathrm{t} 2$ & 0.084034 \\
$\mathrm{t} 3$ & 0.106978 & $\mathrm{t}-\mathrm{iii}$ & 0.106978 & $\mathrm{t} 3$ & 0.106978 \\
$\mathrm{t} 4$ & 0.115000 & & & $\mathrm{t} 4$ & 0.115000 \\
\hline
\end{tabular}

where ' $u$ ' is the displacement in the $x$-direction, and ' $c$ ' is longitudinal strain wave speed given by

$c=\sqrt{\frac{E_{x}}{\rho}}$

Here ' $E_{x}$ ' is the longitudinal tensile modulus and ' $\rho$ ' is the material density. The longitudinal strain in the yarn is given by

$\varepsilon_{x}=\frac{\partial u}{\partial x}$

where the prescribed displacement ' $u$ ' at the pulled end can be described as a function of time by a generalized polynomial as

$u(t)=a t+b t^{2}+c t^{3}+\ldots$

Then for the case of a prescribed linear displacement function with respect to time (constant velocity), we can obtain the longitudinal strain from Eqs. (2), (4) and (5) as

$\varepsilon_{x}=\frac{a}{c}$

where ' $a$ ' is the constant velocity. The reaction force at the clamped yarn end is given by

$F_{x}=\frac{2 E_{x} A}{c} \frac{\partial u}{\partial t}$

where ' $A$ ' is the cross sectional area of the yarn. The reaction force can also be calculated from the impulse-momentum equation which in its generalized form is given by

$m\left(v^{(n-1)}-v^{(n)}\right)=F \Delta t$

where force ' $F$ acting over a short time duration ' $\Delta t$ ' causes a reduction in the velocity ' $v$ ' of travelling mass ' $m$ ' from the previous time step ' $n-1$ ' to the current step ' $n$ '. Through a simple derivation, Eq. (8) leads to an alternate form for the reaction force at the clamped yarn end of Eq. (7) as

$F_{X}=2 \rho A c^{2} \varepsilon_{x}$

The factor of ' 2 ' used in Eqs. (7) and (9) accounts for the arrival and almost immediate reflection of the longitudinal strain wave at the clamped end. If the yarn is considered to be composed of an infinite chain of elements, each element will show a sharp jump in their stress level every time the longitudinal strain wave passes it. The extent of the stress increment depends on the nature of the prescribed displacement at the pulled end. For the constant velocity prescribed displacement condition considered here, the stress increases by a constant increment with each passing of the longitudinal wave. This leads to a stepped formation in the stress plot which is clearly observable from the results. The calculation of longitudinal stress at a particular location ' $x$ ' along the yarn length follows from the calculation of strain as

$\sigma_{x}=\frac{E_{x}}{c} \frac{\partial u}{\partial t}$
The distance between the steps will depend on the location of the element along the yarn length and the corresponding time it takes for one reflection of the longitudinal wave from the point of interest to the pulled end, and also to the fixed end.

The internal energy or elastic strain energy of the yarn is given by

$\mathrm{SE}_{\mathrm{yarn}}=\frac{1}{2} \int_{0}^{L} E_{x} \varepsilon_{x}^{2} A d x$

The kinetic energy of this yarn, held at one end and pulled at the other end with constant velocity, is given by

$\mathrm{KE}_{\mathrm{yarn}}=\frac{1}{2} m v^{2}=\frac{1}{2}(\rho A l)\left(c \varepsilon_{x}\right)^{2}$

where ' $l$ ' is the instantaneous yarn length, i.e. the length of yarn behind the front of the longitudinal strain wave. It is important to note in the above equations that the quantity $\varepsilon_{x}$, and consequently all other terms that depend on it, varies with time depending on the propagation of the longitudinal wave. Thus Eq. (6) actually represents the increment by which the longitudinal strain keeps increasing in a step wise manner for each passing of the longitudinal wave at any fixed location along the yarn length. Fig. 4 compares the results between all cases. As can be observed the new method of modeling yarns with shell elements which is used within the HEA approach closely agrees with the baseline case. There is a good agreement between the yarn internal energy for cases \#1 and \#2, as seen from Fig. 4a. The peak yarn kinetic energy from Fig. 4b for cases \#1 and \#2 closely agrees with the analytical prediction obtained using Eq. (12), with less than a two percent difference when normalized with respect to the analytical prediction. The fluctuation of the kinetic energy between a near zero value and the peak is in accordance with the propagation of the longitudinal wave through the yarn that reflects at the boundaries. Using Eq. (7), the first increment of the reaction force at the clamped end, viz. at the arrival and immediate reflection of the longitudinal wave is $22.1 \mathrm{~N}$. The reaction force grows by this increment with each reflection of the longitudinal wave at the boundary. Comparing the reaction force predictions of cases \#1 and \#2 from Fig. 4c, we see that both cases closely agree with the analytical prediction, with less than half a percent difference when normalized with respect to the analytical prediction. However for all the results, we see that cases \#3 and \#4 differ from the baseline results. Fig. $4 \mathrm{~d}$ compares the error in predictions of cases \#3 and \#4 with respect to the analytical predictions. Both the magnitude and percentage of error in the reaction force at the fixed end and the time instant at which the longitudinal wave reflects from the fixed end have been shown. Because of the smaller longitudinal wave speed in case \#4 caused by the increased yarn density, the peaks are shifted to the right and lag behind the other cases. Consequently the error in both the reaction force at the fixed end and the time instant at which the wave reaches the fixed end grows in magnitude with each wave reflection at the fixed end. For case \#3, the magnitudes of the peak reaction force and peak kinetic energy are smaller than the baseline case because of the lost yarn mass, even though the time instant of occurrence matches the baseline case since the longitudinal wave speed remains the same. Therefore Fig. 4d does not display the error in time for case \#3. The error in the predictions of the reaction force of case \#3 grows in magnitude with each reflection at the fixed end as can be seen from Fig. 4c. There is an approximately 6.5\% error in the reaction force predictions of case \#4 with respect to the analytical predictions, while that of case \#3 is approximately $12.2 \%$. This supports the conclusion that the new method of modeling yarns with shell elements is more accurate compared to the previous 

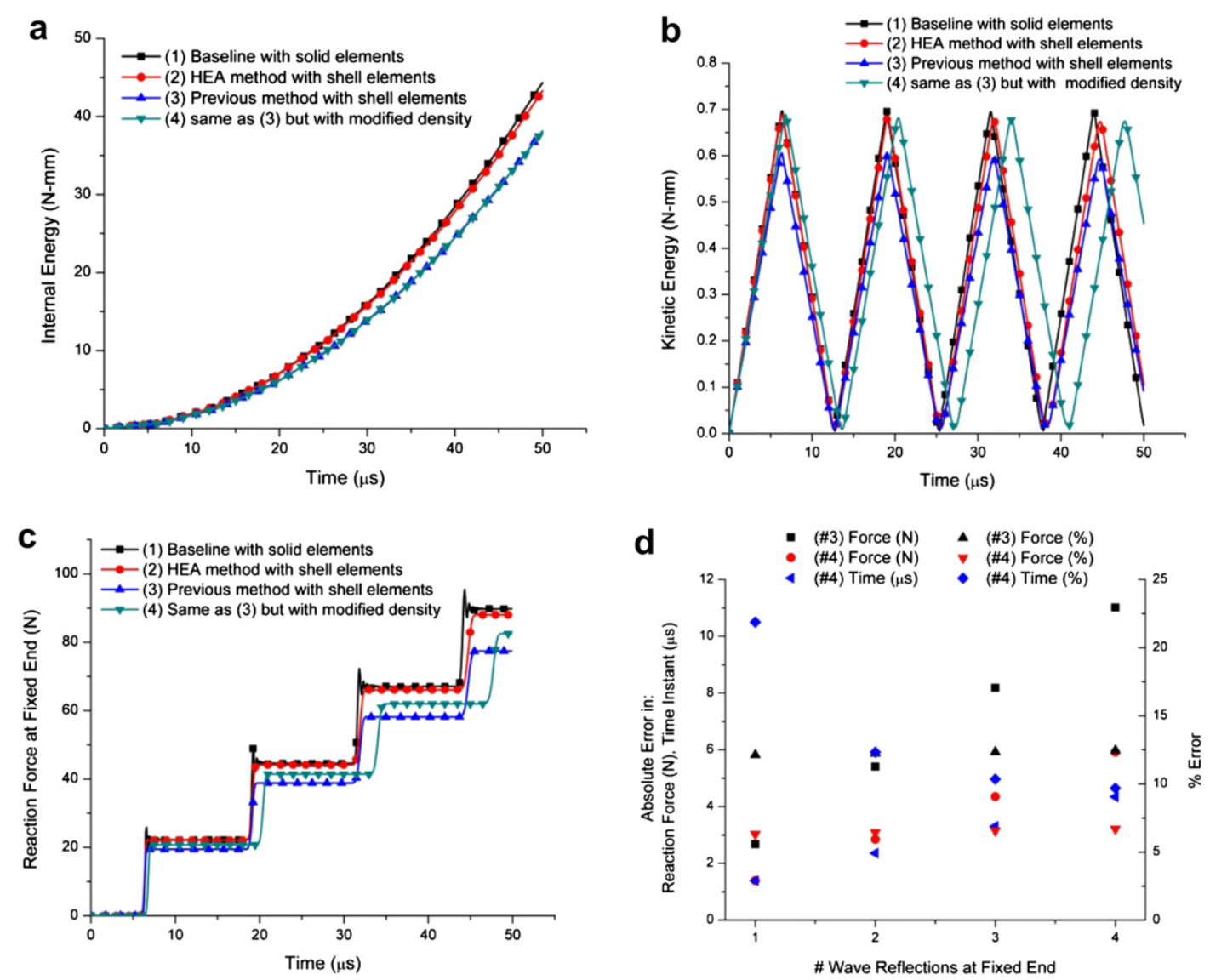

Fig. 4. Comparison of results: (a) yarn internal energy, (b) yarn kinetic energy, (c) reaction force at clamped end, (d) error in the predictions.

method with thickness jumps, and that even modifying the yarn material density in an attempt to conserve mass leads to incorrect results. An additional advantage is that the new method also better represents the yarn cross section. As we will see in Section 5 , these shell elements used to model the yarns find use in regions away from the impact zone where the response is predominantly tensile in nature and there are lesser extents of inter yarn interactions such as sliding and reorientation, making it an important requirement that the tensile response is accurately captured. We have just demonstrated through these tensile test simulations that this new method of modeling yarns with shell elements is capable of doing exactly that.

\section{Hybrid element analysis applied to a single yarn}

When choosing between solid and shell elements, there is a tradeoff between accuracy and computational expense. Therefore a good approach would be to combine the benefits of both finite elements into the yarn model. At the impact zone a higher level of accuracy is needed as there are increased projectile-curvature and transverse effects such as transverse yarn compression, shearing, and bending which are primarily due to projectile-yarn interactions. At locations removed from this impact zone, there are much lesser extents of projectile-fabric and yarn-yarn interactions. The main mode of yarn deformation is tensile in nature which can be modeled accurately using a lower level of resolution. In this region the emphasis is therefore on computational efficiency, so that large yarn dimensions can be modeled. Further, as seen in experimental transverse impact testing of yarns and fabrics, the predominant failure is always underneath the projectile. A useful approach then would be to use solid elements to model the yarns at the impact zone and shell elements at regions away from the impact zone. We refer to this approach as the hybrid element analysis and define it as 'the finite element analysis of a structure by combining different finite element formulations at both a single and multiple scales of modeling'. For example, the yarn is considered as a single scale model when a homogenized approach is used so that filament level architecture is not considered. While modeling a fabric, multiple scales of modeling would imply modeling using both yarn level resolution and a homogenized membrane type assumption for far field regions. In this study, we apply the HEA method to a single scale of modeling since we only consider a yarn level architecture. Future work will present the HEA method applied to multiple scales of modeling. Fig. 5 displays the HEA method applied to a single yarn. The solid elements are located around the center of the yarn which is impacted, and is surrounded on either side by shell elements. A tied interface is used between the two types of finite elements. If an even number of solid elements is used through the thickness, it is also possible for the solid and shell elements to share common nodes at the interface. In such a case, the element formulation of the solid elements must support nodal rotations which will ensure compatibility with the degrees of freedom of the shell elements. Since the material properties used in the material models for both the solid and shell elements are the same, and the yarn cross sectional area is the same by virtue of our new modeling approach for yarns modeled using shell elements, there is a matching of impedances across the interface. This is important as it ensures that there are no reflections of the longitudinal strain wave at the interface, and that the transverse displacement or bending wave propagates properly across the interface. 

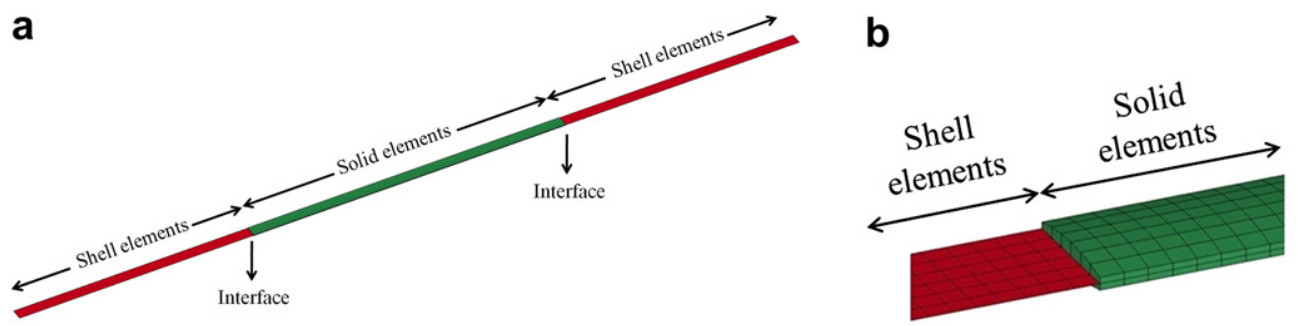

Fig. 5. (a) HEA of a yarn using shells and solids (top view). (b) Close up at the interface.

\section{Impedance matching in the HEA approach}

Impedance matching is a crucial requirement especially in multi scale models that contain interfaces. Failure to match impedances across interfaces can introduce significant errors in the analysis due to interfacial wave reflections, especially in wave dominated high rate impact phenomena such as the transverse impact of woven fabrics. This was seen in the multiscale fabric model of Barauskas and Abraitiene [13,14] where yarn failure had to be artificially 'turned off for the shell yarn region adjacent to the membrane region. This was attributed to the possible overestimation by LSDYNA of local strains in the finely meshed yarn region adjacent to the coarsely meshed membrane region, however an impedance mismatch between the two reasons was more likely the primary cause of the premature yarn failure, due a stress buildup caused by interfacial reflections of the longitudinal wave, which then necessitated the removal of the failure criterion. To demonstrate that there are no reflections of the longitudinal strain wave at the shell-solid interface using the HEA approach, and to highlight the detrimental effects produced by an artificial modeling-induced impedance mismatch at the interface, four test cases are set up using the test case parameters and yarn material properties as before, and simulated using the dynamic finite element code LSDYNA. In the first test case, entitled 'solid', the yarn is modeled using solid elements and this serves as the baseline. In the second test case entitled 'HEA', the HEA approach as outlined earlier is used, see Fig. 5a. The central portion of the yarn is modeled with solid elements and measures $20 \mathrm{~mm}$ while shell elements are used on either side with each shell element region measuring $15 \mathrm{~mm}$. In the third test case, entitled 'impedance mismatch \#1 (IM1)' the density of the yarn in the shell element region has been increased by 1.5 times, from its original value of $1440 \mathrm{~kg} / \mathrm{m}^{3}$ to $2160 \mathrm{~kg} / \mathrm{m}^{3}$. In the fourth test case, entitled 'impedance mismatch \#2 (IM2)' the cross sectional area $(A)$ of the yarn in the shell element region has been increased by 1.5 times, from its original value of $0.0512 \mathrm{~mm}^{2}$ to $0.0760 \mathrm{~mm}^{2}$. This dynamic tensile test is dominated by the tension generated in the yarn due to the longitudinal elastic modulus. The inertial effects are not significant as evident by the yarn kinetic energy which is negligible compared to the yarn internal energy. Further, a constant velocity is prescribed at the pulled end. Thus even though the mass of the yarn changes as we are changing the yarn material density and cross sectional area, this process allows us to isolate the effect the associated impedance mismatch will have on the system response for the purpose of illustration. The total acoustic impedance is given by

$z=\rho c A$

In the third test case, by changing the yarn density of the shell element region, the corresponding longitudinal strain wave speed will change according to Eq. (3). However in the fourth test case, the longitudinal strain wave speed in both the solid and shell element region will remain the same. Fig. 6 displays contours of the longitudinal tensile stress in the yarn during the first $7 \mu$ s, during which time the wave travels from the pulled to the fixed end and reflects back towards the pulled end. The illustrated labels beside each yarn in Fig. 6 correspond to 'test case - time instant $(\mu \mathrm{s})$ '. As can be seen, the results of the HEA case closely follow the baseline 3-d case. The longitudinal tensile stress developed behind the wave front is constant as indicated by the solid color band in the plots. On its way to the fixed end, the longitudinal wave passes through two shell-solid interfaces, and as is observed from the first two test cases, there are no wave reflections at the interfaces. In test case IM1, the longitudinal wave lags behind the other cases as the yarn density has been increased in the shell element region. The longitudinal waves for the cases solid, HEA, and IM2 reach the first shell-solid interface at around $2 \mu$ s. Upon close examination of the plots labeled IM1-3 and IM1-4, the reflection of the longitudinal wave at the first shell-solid interface is apparent as the yarn stress contour plot no longer remains a single solid color behind the wave front, which would have represented a constant stress, rather there is a band of stresses present as indicated by the different band of colors. Similarly, the plots labeled IM2-3 and IM2-4 also exhibit a reflection of the longitudinal wave at the interface. Upon reaching the pulled end, this reflected longitudinal wave reflects once more and heads towards the fixed end, where it will meet the original longitudinal wave which is on its way back from the fixed end. In addition to this, there are also reflections that will occur at the second shell-solid interface. This leads to a non-uniform state of stress within the yarn as seen in the final plots labeled IM1-7 and IM2-7 where a discrete band of colors is observed throughout the yarn length. This process continues during the entire dynamic tensile test. However as seen in the plots labeled solid-7 and HEA-7, the stress remains uniform behind the front of the longitudinal wave as indicated by the single solid color band. Fig. 7 helps quantify the differences in the responses seen in Fig. 6. Fig. 7a compares the longitudinal tensile stress developed at a location $12.7 \mathrm{~mm}$ from the right pulled end, which lies within the shell element region. The shape of the stress history plot for both the baseline and HEA cases follows a uniform stepped shape with the stress level remaining constant between the passing of the wave and the next arrival of the reflected longitudinal strain wave from the boundaries, which is accompanied by a sharp incremental rise in the stress level. However as is evident from Fig. 7a, during the time between which the longitudinal wave has passed through a location and when it arrives again at that location after a reflection from a boundary, for both the IM1 and IM2 cases, the stress level does not remain constant and fluctuates with sudden rises and dips indicative of the reflections from the interfaces. This causes the earlier mentioned discrete band of colors in the stress contour plots, seen in Fig. 6. Such an effect is detrimental as it can either delay or cause premature yarn failure.

As expected, the baseline and HEA cases predict the same stress levels, which for further validation have been compared with the analytical predictions based on 1-d wave propagation theory, as outlined earlier. For our particular test case, substituting the yarn material properties and prescribed velocity at the pulled end into 


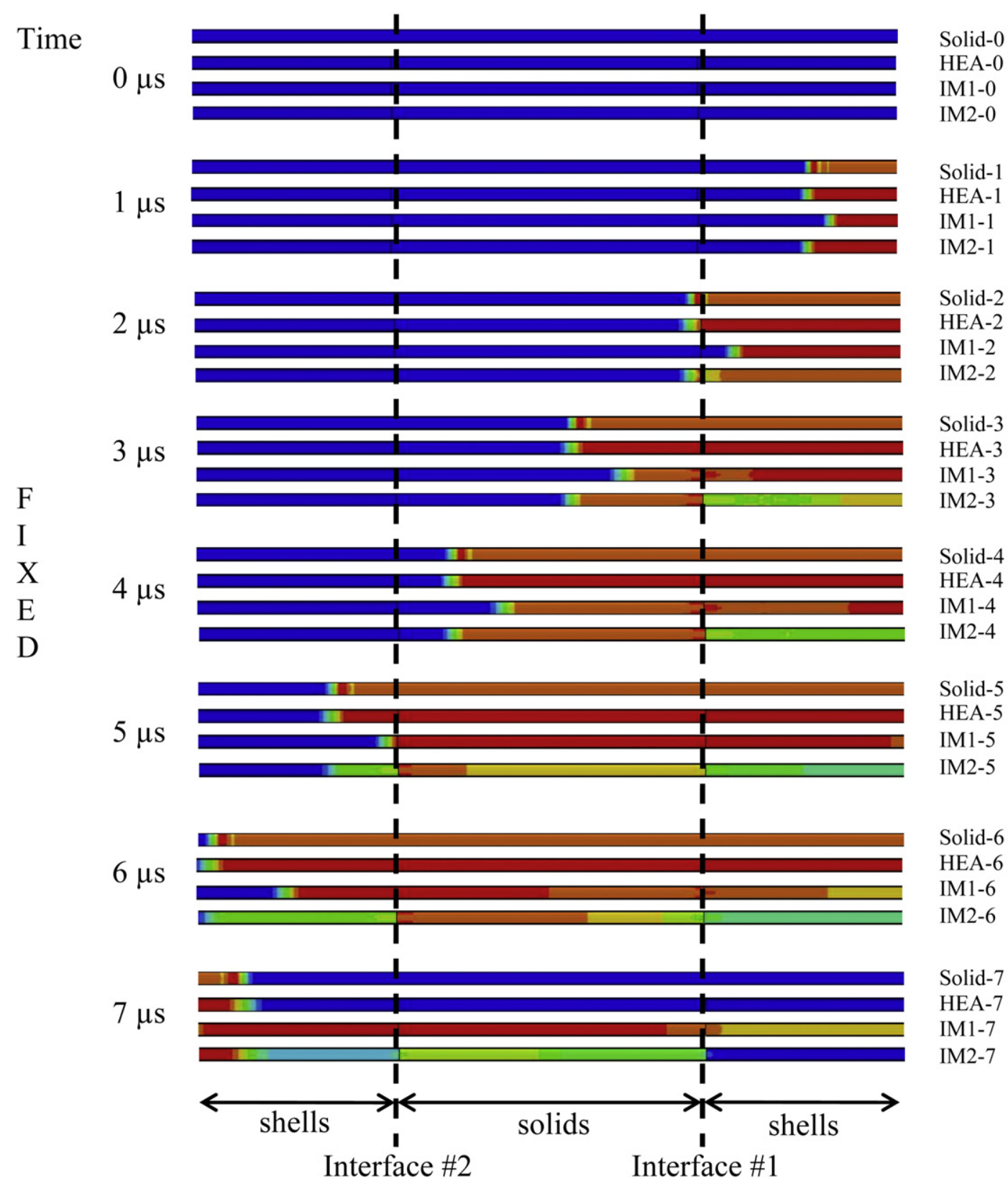

Fig. 6. Longitudinal wave propagation through the yarn.

Eq. (10), we obtain the first stress increment as 198.1 MPa. This stress value, as well as the following stress increments, closely agrees with the baseline and HEA results with less than a percent difference when normalized with respect to the analytical prediction. However as seen from the IM1 and IM2 cases in Fig. 7a, the multiple reflections at the two shell-solid interfaces cause a fluctuation and therefore an incorrect prediction of the longitudinal stresses. This error in predictions grows with time and even exceeds $30 \%$ at around $18 \mu$ s for IM2 with respect to the analytical prediction. Fig. $7 \mathrm{~b}$ compares the reaction force at the clamped end of the yarn. Substituting the yarn material properties and prescribed velocity at the pulled end into Eq. (7), we obtain the first reaction force increment as $20.3 \mathrm{~N}$ which closely agrees with both the baseline and HEA cases, again with less than a percent difference when normalized with respect to the analytical prediction. However the IM1 and IM2 cases show a fluctuation in the predictions, with the error once again increasing with time.
Thus it becomes important to match impedances across any interfaces present within the FE model. As we will observe in Sections 6 and 8, yarn failure is implemented using a stress-based failure criterion in LS-DYNA. Whether the failure model is stress- or strain-based, it becomes all the more important that impedances are matched across the interfaces so that the model predicts the correct stress state, which otherwise could lead to premature yarn failure because of a stress buildup caused by multiple reflections from a fictitious interface.

\section{Results and discussion of single yarn modeling}

Consider the case of a $0.5 \mathrm{~g}$ wedge shaped rigid projectile transversely impacting a $50 \mathrm{~mm}$ long uncrimped yarn at its center at $200 \mathrm{~m} / \mathrm{s}$. The projectile moves along the $y$-direction. The yarn is gripped at both ends. The material properties of the yarn remain the same as before. Two sets of simulations have been set up using 

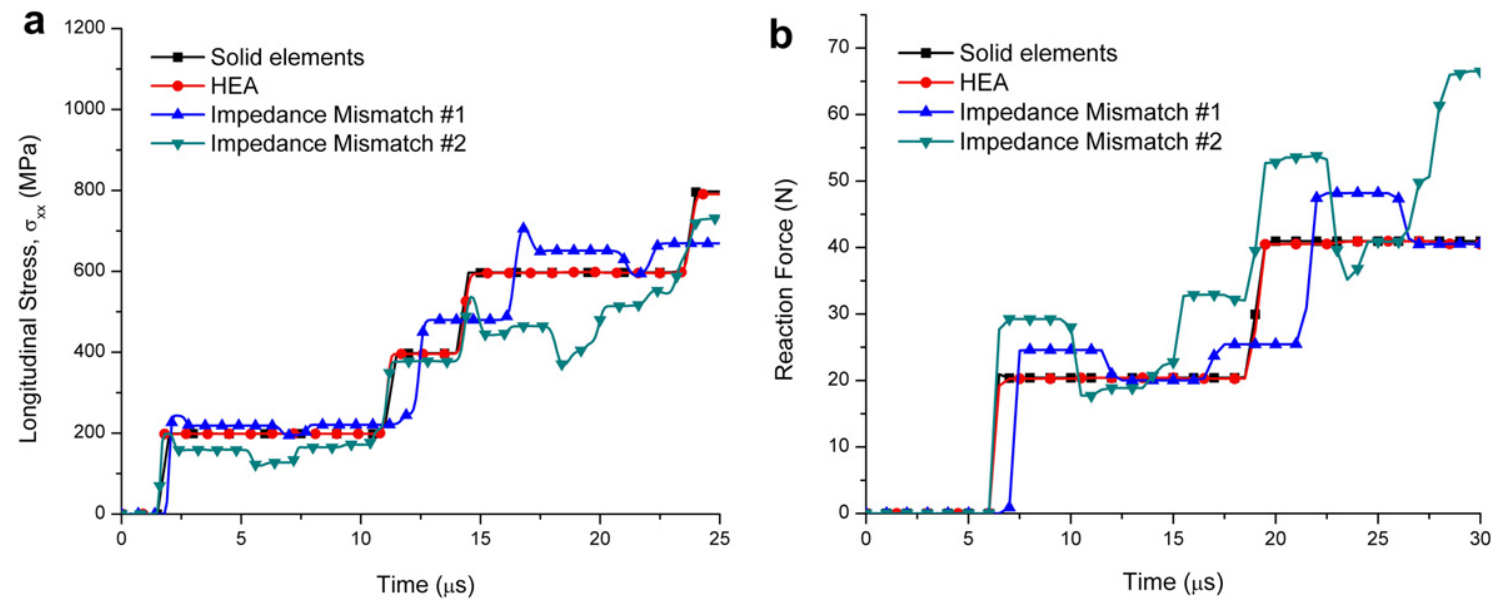

Fig. 7. (a) Longitudinal tensile stress at $x=12.7 \mathrm{~mm}$ from the pulled end. (b) Reaction force at the fixed end.

the dynamic finite element code LS-DYNA. In the first set, the yarns are modeled using (a) shell elements, and (b) solid elements. The complexity of failure is not considered in this set of simulations that focuses on yarn deformations. This set of simulations allows us to compare the initial response of the shell element case to the solid element case. In the second set of simulations, the yarns are modeled using (a) solid elements, and (b) the HEA approach as outlined earlier. Failure is implemented for this set using an element erosion failure model with a maximum principal stress failure criterion of $2759 \mathrm{MPa}$.

Fig. 8 compares the results from the first set of simulations. From Fig. 8a, we observe the velocity agrees in both cases; however the yarn internal energy grows at a faster rate from the moment of impact onwards for the shell element case and the yarn kinetic
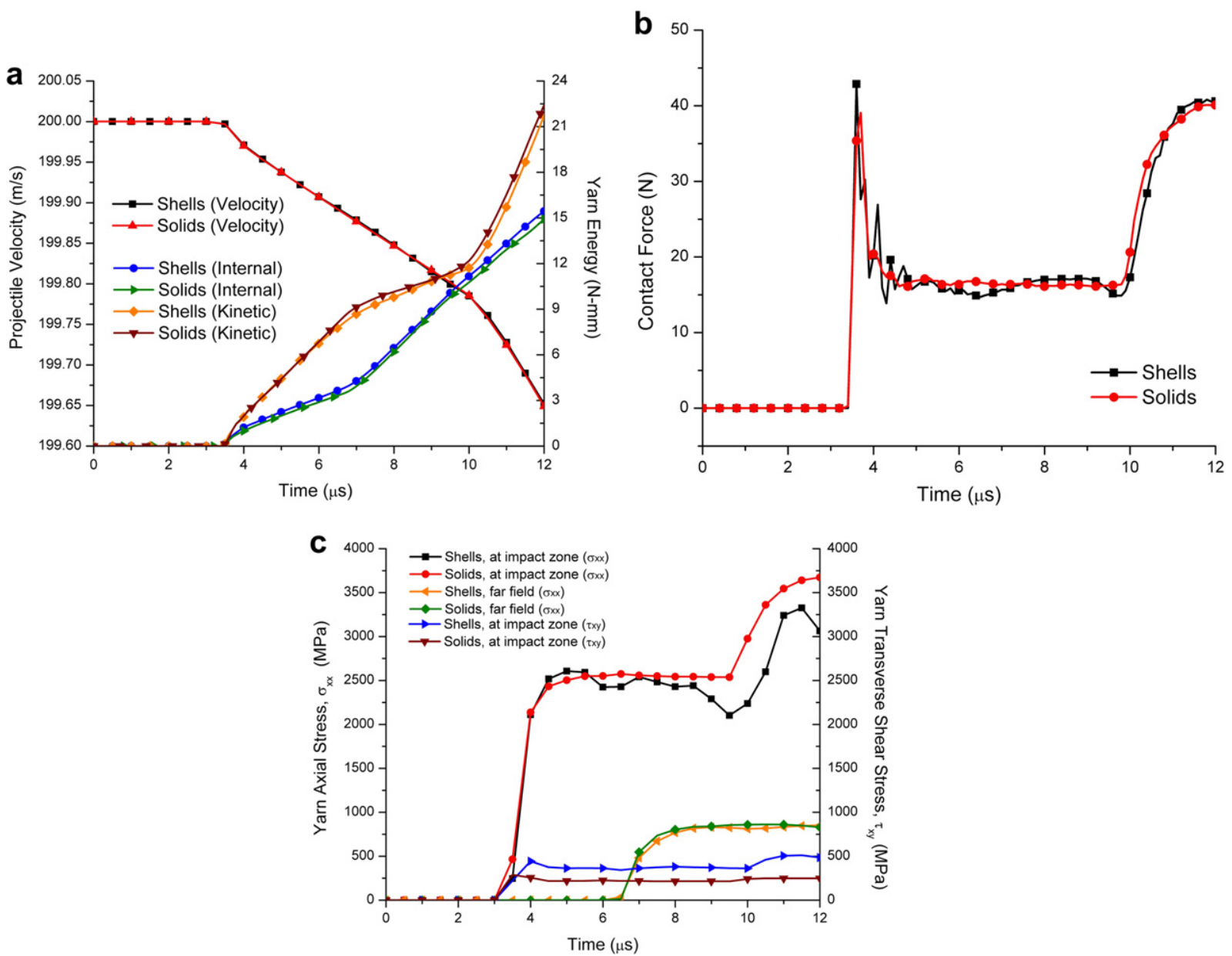

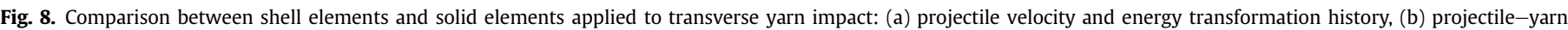
contact force history, (c) yarn stress history. 

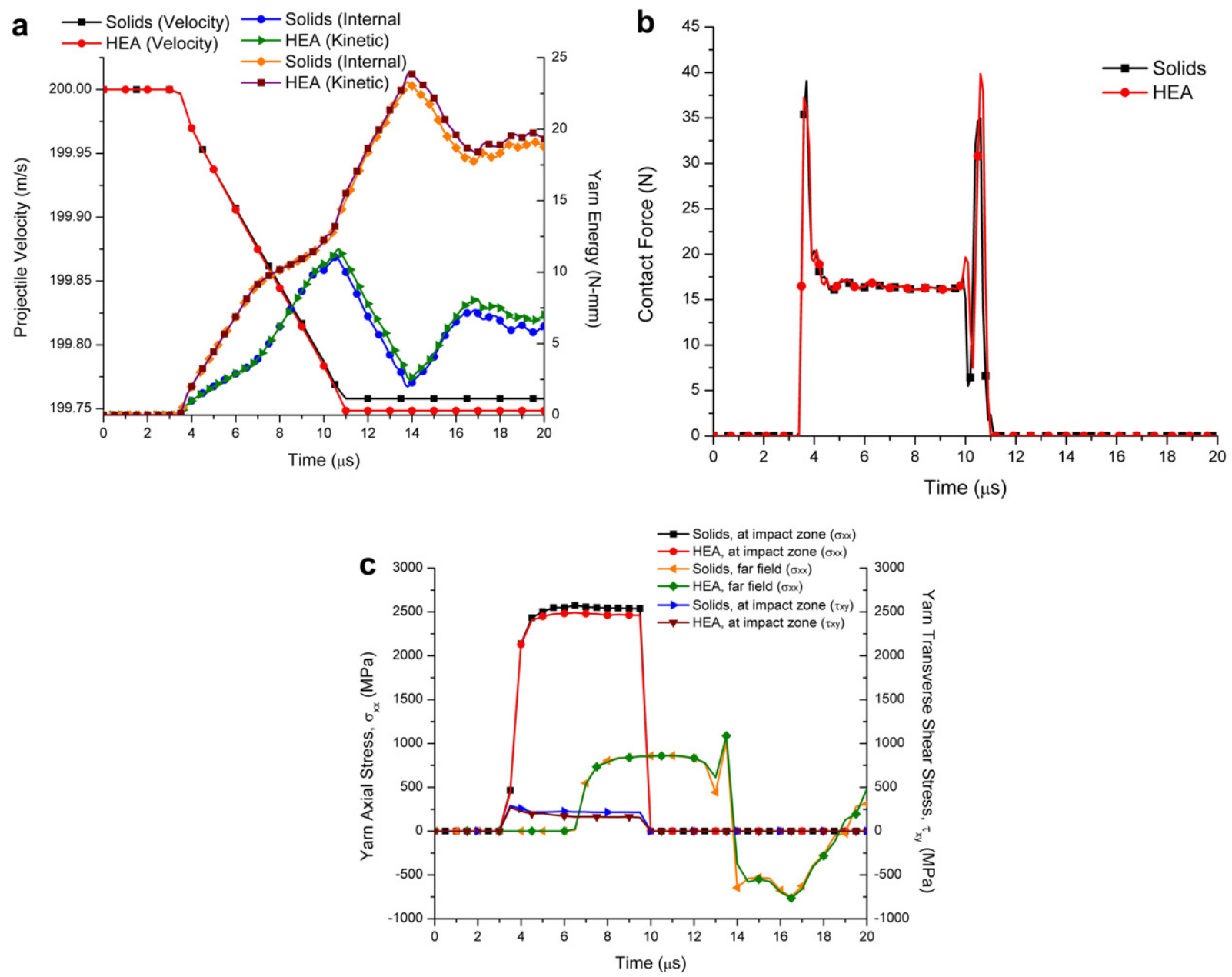

Fig. 9. Comparison between solid elements and HEA applied to transverse yarn impact: (a) projectile velocity and energy transformation history, (b) projectile-yarn contact force history, (c) yarn stress history.

energy begins to deviate slightly from the solid element case after $6 \mu$ s. Fig. 8b compares the total projectile to yarn contact force. This contact force is an indication of the tension generated in the yarn. The vertical component of the tension developed within the yarn is

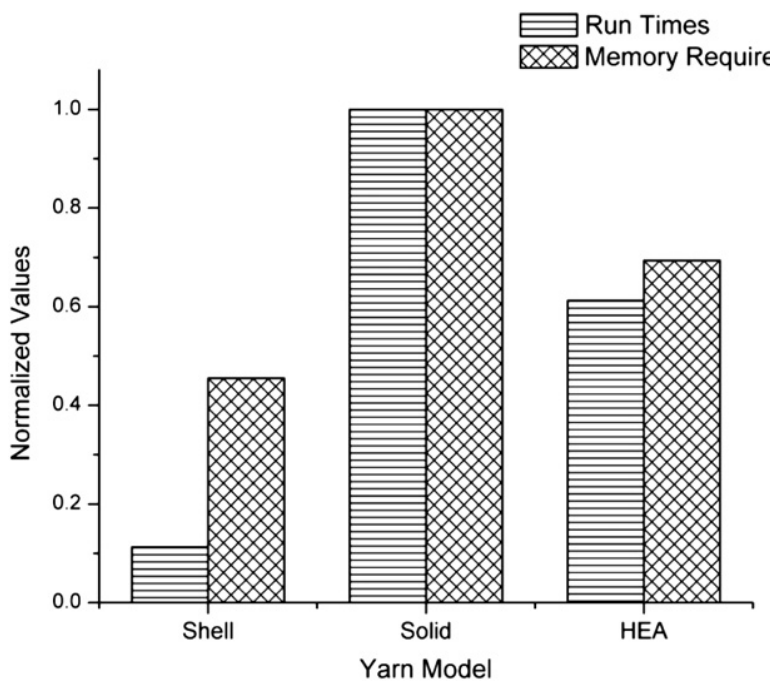

Fig. 10. Computational requirements. related to the decrease in projectile velocity through the impulse-momentum equation. While Fig. 8a and b implies similar yarn tensile forces are developing in both cases, it is not a sufficient measure of the ability of the shell elements to be used for yarn modeling at the impact zone. For this, we turn to Fig. $8 \mathrm{c}$ which compares the stress distributions in the yarn at two locations. The longitudinal tensile stress $\left(\sigma_{x}\right)$ and transverse shear stress $\left(\tau_{x y}\right)$ have been measured near the bottom surface of the yarn directly under the impact location of the projectile. In addition, the longitudinal tensile stress is also measured at a far field location that corresponds to $1 \mathrm{~mm}$ from the right gripped end. We observe that the stress predictions from the shell element case differ from the solid element case at the impact zone. The shell element formulation used here, as in most commercial FE codes, is based on a first order shear deformation theory. This implies the transverse shear strains are constant through the thickness. For isotropic materials, a shear correction factor of 5/6 is used in an attempt to correct this issue, that leads to a violation of the zero traction condition at the top and bottom surfaces of the shell. More importantly, the shell elements are unable to account for any thickness changes in the yarn cross section due to compression by the projectile. The maximum interaction is seen at the impact zone where the projectile compresses and shears the yarns at very high velocity. In addition the curvature of the projectile induces high flexural and shear stresses in the yarn around the impact zone. Here momentum transfer occurs over very short time intervals and is limited to the 
a

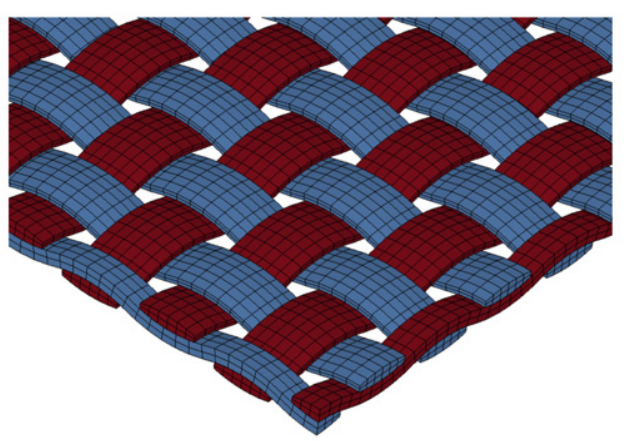

b

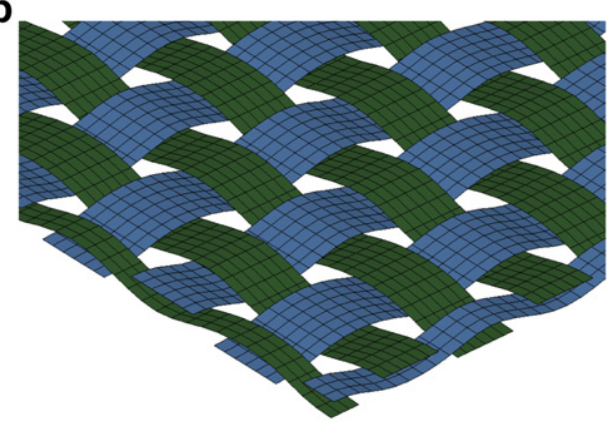

Fig. 11. FE model of a plain weave fabric using only (a) solid elements, (b) shell elements.

region within the front of the longitudinal stress wave. The difference in the stress predictions at the impact zone demonstrates the inability of the shell elements to properly model these interactions, more so as this is also the region where yarn failure would occur during an impact test. However at far field regions, the deformation of the yarn is predominantly tensile in nature. We observe a good agreement in the stress predictions at the far field region. This supports the reasoning of using solid elements at the impact zone where higher accuracy is required, and shell elements at far field regions where the emphasis is on computational efficiency.

Fig. 9 compares the results from the second set of simulations. The simulation run time has been increased from $12 \mu \mathrm{s}$ to $20 \mu \mathrm{s}$ with failure now implemented. From Fig. 9a we observe an excellent agreement between both cases prior to yarn failure. The first instant of element failure for the solid element case is $9.87 \mu \mathrm{s}$ and is $9.95 \mu \mathrm{s}$ for the HEA case. After the yarn snaps, the yarn internal energy rapidly drops accompanied by an increase in the yarn kinetic energy as the yarn flaps freely. The projectile velocity now remains constant as it has completely penetrated through the yarn. The post-failure response displays a similar trend in both cases with a minor difference in the yarn energies. Fig. 9b compares the projectile-yarn contact force and shows a good agreement between both cases. In Fig. 8c we had observed a difference in stress predictions at the impact zone, however as now seen in Fig. 9c, there is a good agreement between the stress predictions at both the impact zone and far field regions.

Fig. 10 compares the computational requirements between the three cases in terms of simulation run times and memory requirements. It is important to consider the memory requirements in addition to the run times. High performance clusters such as the Beowulf cluster are created by combining many similar multiprocessor nodes together. However, typically the memory available per node ranges from 2 to $8 \mathrm{~GB}$. If the simulation initialization phase in LS-DYNA requires more memory than that available to a single processor or node, it will not run regardless of the total memory available in the cluster. This problem of limited memory available per node is not an issue in high performance systems or workstations that use a globally shared type of memory architecture wherein all processors have access to all the memory. Such systems typically have a memory in the range of 32-64 GB. However the cost of such systems usually far exceeds that of a high speed cluster of similar configuration.

The shell element case has the best computational efficiency, but for reasons stated earlier, cannot be used for regions underneath the projectile at the impact zone. The HEA case runs almost $40 \%$ faster than the solid element case. This unique HEA approach has resulted in reproducing the predictions of the baseline numerical model but at a fraction of the computational expense.
The ratio of lengths of the solid element portion to shell element portion in this particular HEA case was 0.66 , and by reducing this ratio, the computational efficiency can be further increased. However there is a limit to the extent by which the solid element region can be reduced. For example in this particular impact case,
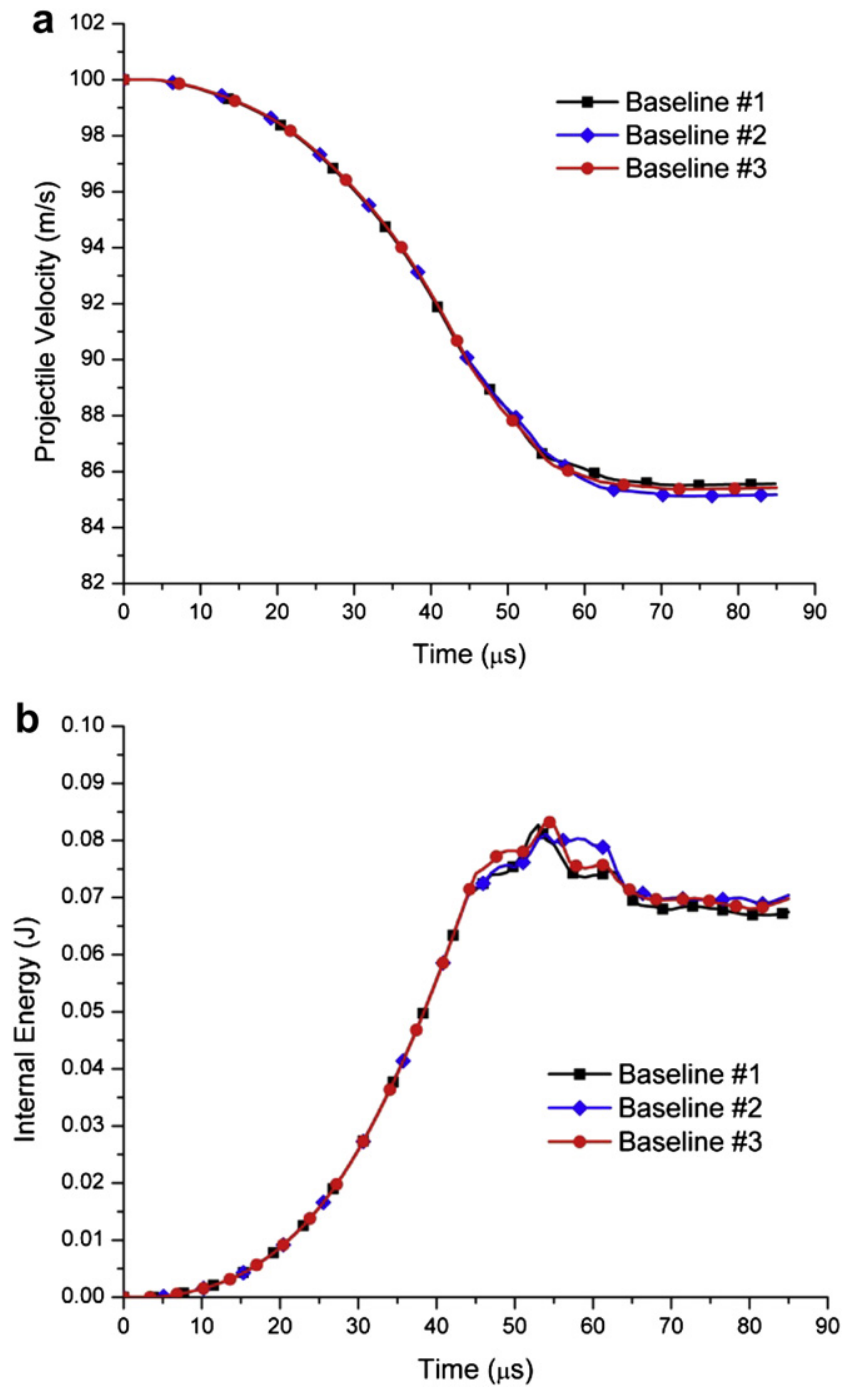

Fig. 12. Comparison between baseline models for $100 \mathrm{~m} / \mathrm{s}$ impact velocity: (a) projectile velocity history, (b) internal energy history. LS-DYNA contact algorithm for baseline \#1 (type 26), baseline \#2 (type a3), baseline \#3 (type 4). 
b

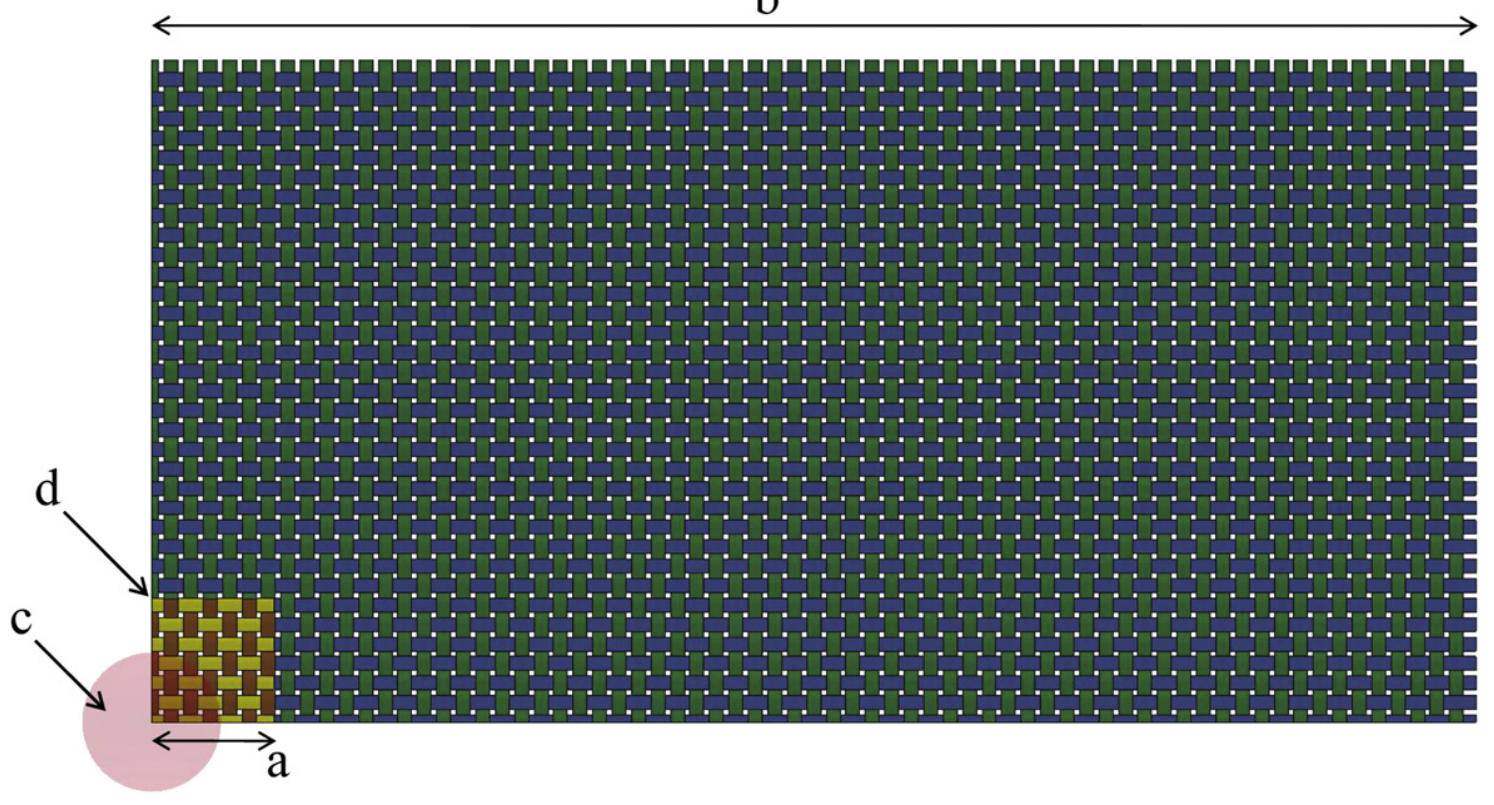

Fig. 13. Setup of a fabric model using a single scale HEA approach: (a) yarns - solid elements, (b) yarns - shell elements, (c) projectile, (d) solid-shell interface.

from a series of simulations we observed that the minimum size of the solid element portion must be at least two to three times the maximum dimension of the projectile's impact face. For dimensions less than this, yarn failure was observed at the shell-solid interface immediately following impact.

\section{Single scale modeling of plain weave fabric systems and setup of the baseline numerical models}

At this stage, the HEA approach has been validated for a single yarn model by comparing it to both a baseline yarn FE model as well as analytical predictions. The next stage is to extend the HEA approach to a fabric FE model comprised of these yarns. This stage begins with first setting up the baseline fabric model which will be used for comparison to the HEA fabric model. As mentioned earlier, a single scale model of a plain weave fabric system refers to the approach where one level of resolution is used throughout the model. The fabric may be entirely modeled by explicitly capturing the yarn architecture, with the use of only solid elements [19], shell elements [16], or 1-d elements [21]. The fabric may also be entirely modeled by homogenizing the yarns into a single and continuous membrane type layer, using only shell elements [3,5]. There is no mixing of macro and micro levels of architecture in such a modeling approach. Fig. 11a displays the FE model of a single layer of a plain weave fabric modeled using only solid elements and maintaining a yarn level resolution. Fig. 11b displays an equivalent model using only shell elements, which uses our new method of non-uniform nodal thicknesses to capture the yarn geometry. Due to symmetry, only one quarter of the fabric needs to be modeled. The baseline fabric numerical model as seen in Fig. 11a is set up using the preprocessor DYNAFAB [17].

Consider the case of a $0.63 \mathrm{~g}$ rigid spherical projectile of diameter $5.55 \mathrm{~mm}$ impacting a $101.6 \mathrm{~mm} \times 50.8 \mathrm{~mm}$ balanced plain weave fabric at the center. The projectile moves along the $y$ direction. The fabric is gripped on all four sides. The material properties of the yarns used in the simulation are: longitudinal elastic modulus $\left(E_{x}\right)$ of $62 \mathrm{GPa}$, transverse elastic moduli $\left(E_{y}\right)$ and $\left(E_{z}\right)$ of $620 \mathrm{MPa}$, and a maximum principal stress to failure of
$3400 \mathrm{MPa}$. The yarns are modeled using single integration point solid elements and the projectile is modeled using fully integrated shell elements. Due to symmetry only one quarter of the fabric has been modeled. A static frictional coefficient of 0.18 is specified for the yarn to yarn contact algorithms and 0.18 for the projectile to yarn contact algorithms. The thickness of the warp and fill yarns is $0.115 \mathrm{~mm}$ and the total fabric thickness at the cross-over locations is $0.23 \mathrm{~mm}$. The yarn count in both warp and fill directions is 34 yarns per inch. Two impact velocities of $100 \mathrm{~m} / \mathrm{s}$ and $200 \mathrm{~m} / \mathrm{s}$ are selected.

One of the most challenging tasks during the finite element analysis of fabric impact is to accurately account for the highly complex projectile-yarn and yarn-yarn interactions by virtue of the complex fabric architecture. This is accomplished through contact algorithms in the FE code that prevent interpenetrations of nodes and segments by applying forces between penetrating nodes and segments. Depending on the contact algorithm used, the manner in which the detection and removal of interpenetrations is accomplished, determination of contact and sliding energies, as

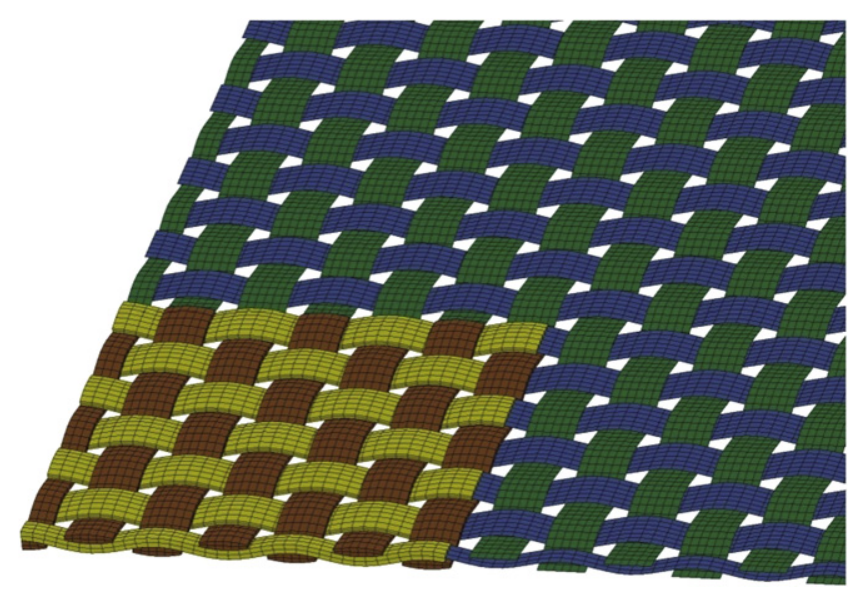

Fig. 14. Close up at the impact region. 
a
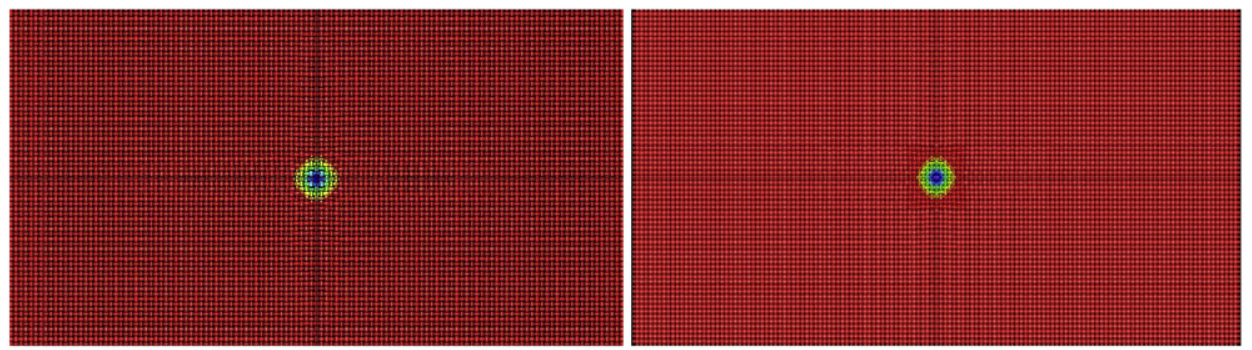

b
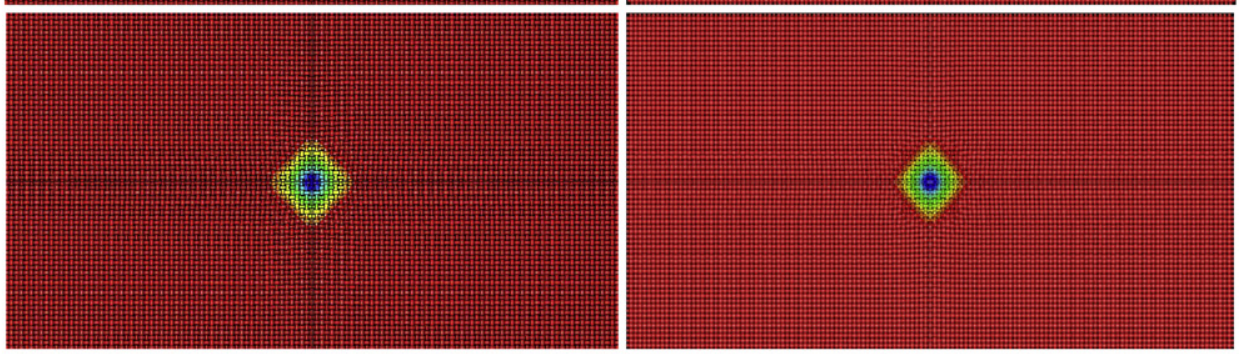

C
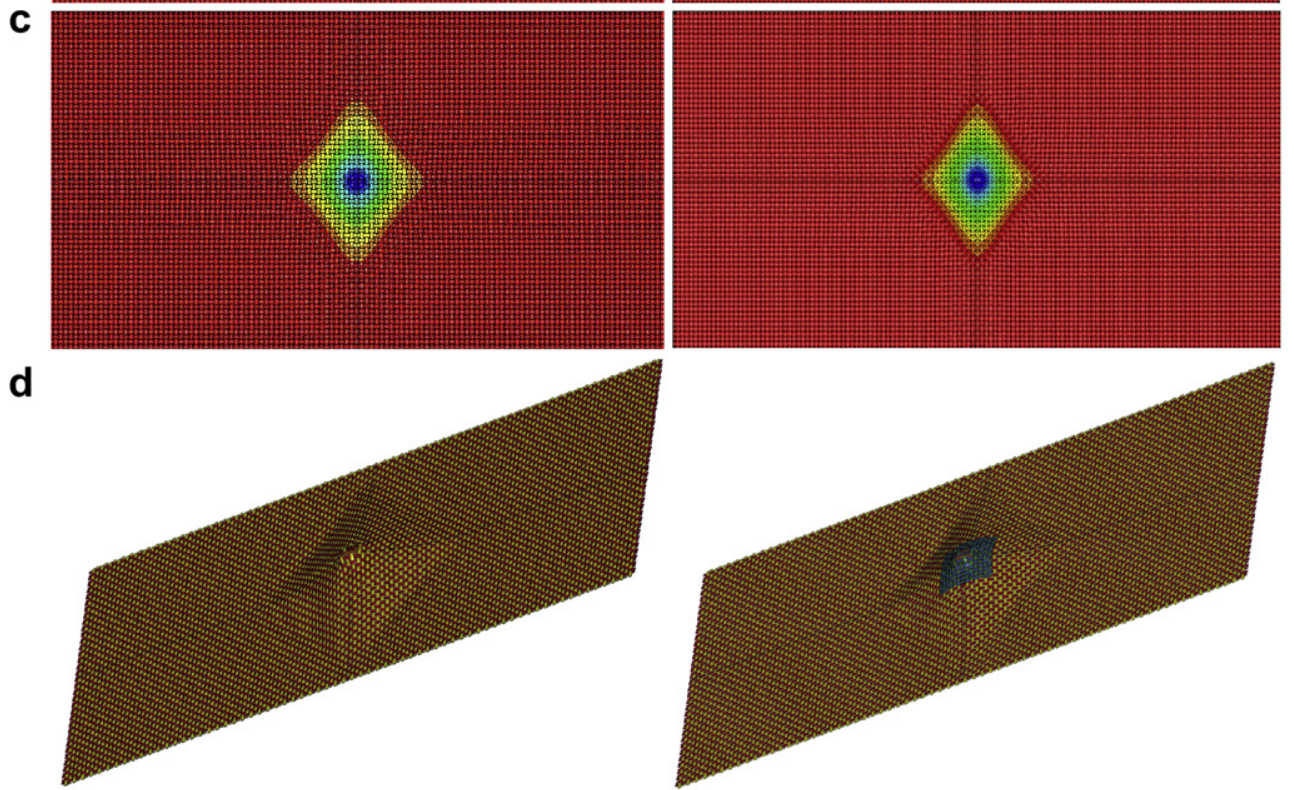

e
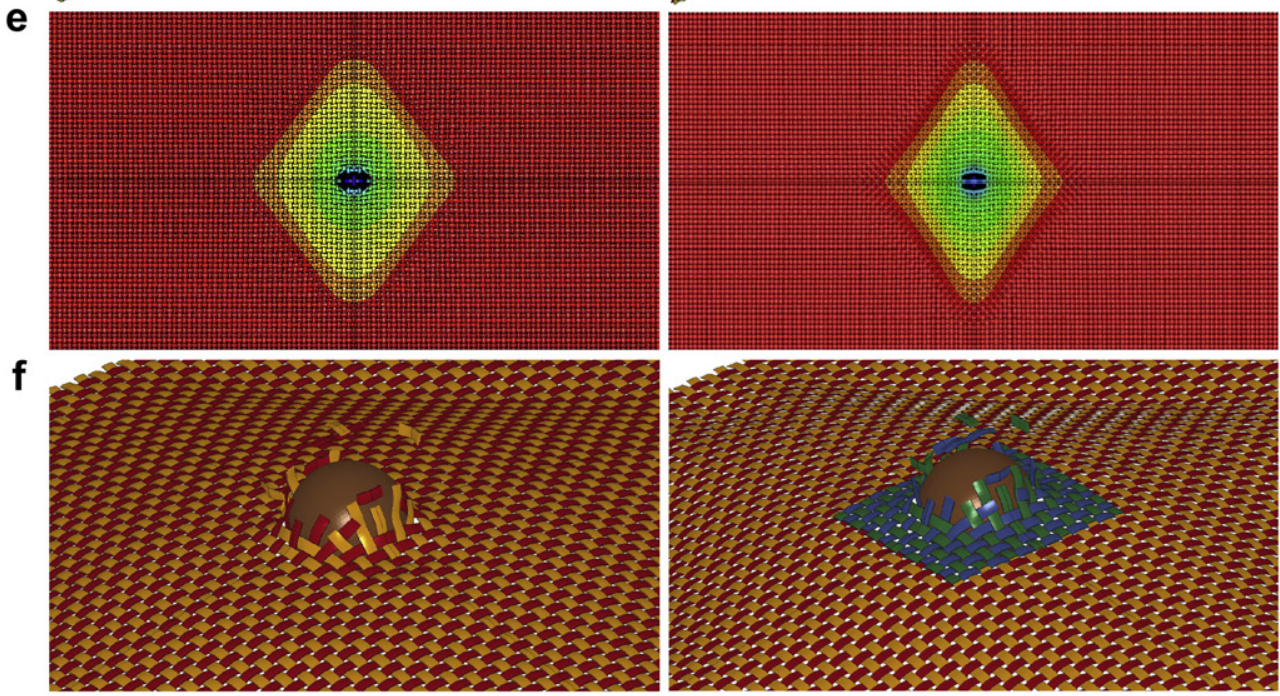

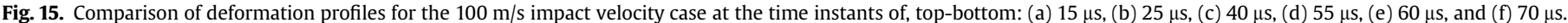
(Left) Baseline \#1, (right) HEA. 

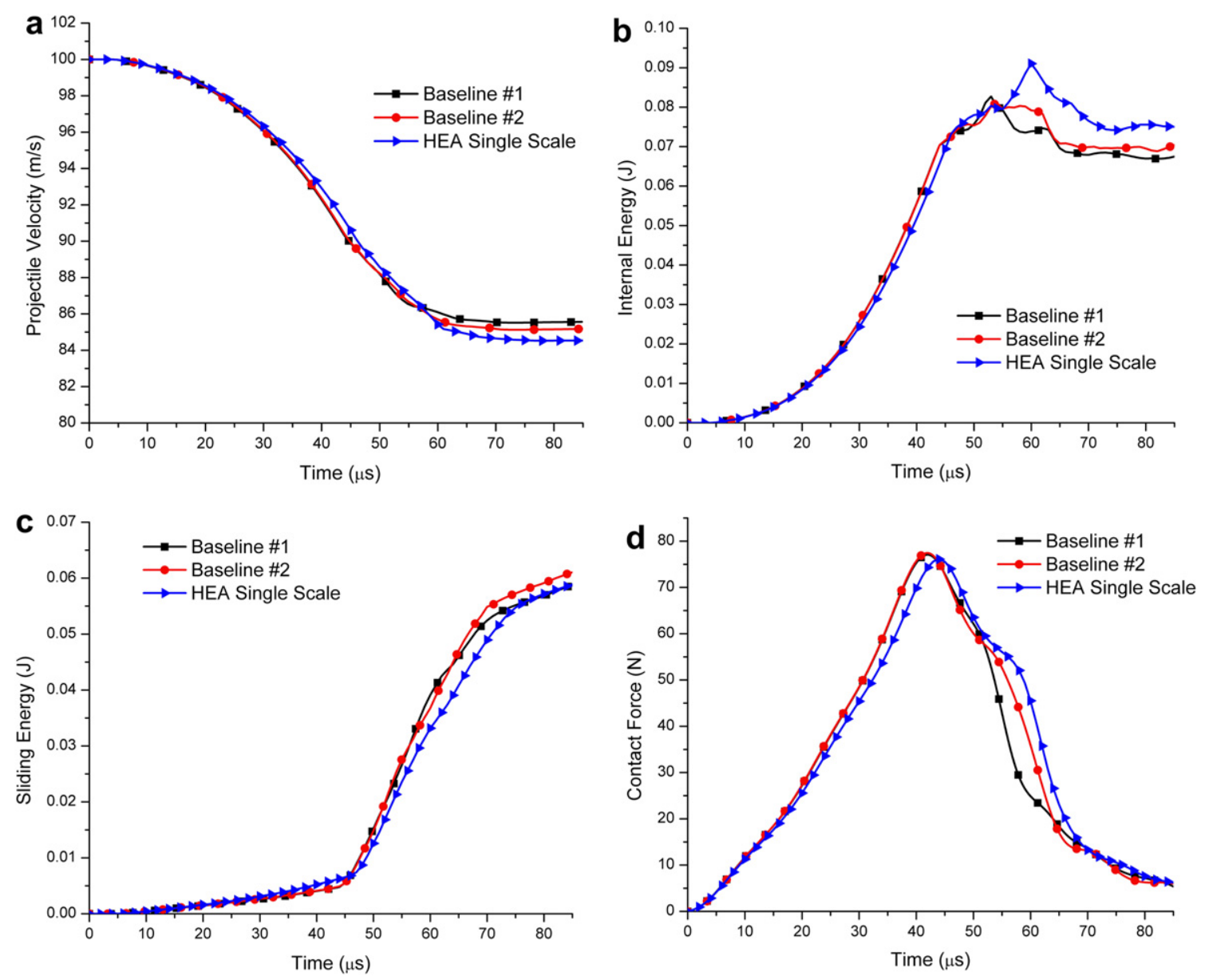

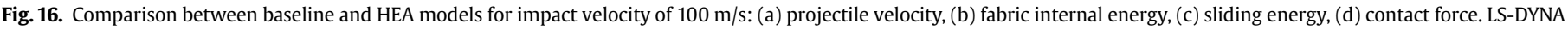
contact algorithm for baseline \#1 (type 26), baseline \#2 (type a3).

well as the transfer of boundaries between interacting segments vary. Consequently with the use of different contact algorithms, the system response and computational run times for the same physical problem may differ either negligibly or significantly. It is therefore important to investigate this effect and accordingly three baseline simulations are set up using LS-DYNA contact types 26, a3, and 4 respectively. Further details of the implementation and working of these contact algorithms can be obtained from Ref. [22]. The first baseline uses the most robust contact algorithm of the three and consequently is the most computationally intensive, while the second baseline uses the least computationally intensive algorithm for this type of problem. The first and third baselines use a single surface type contact algorithm which ensures that no two adjacent yarns (viz. warp-warp or fill-fill) can penetrate each other. The second baseline uses a master and slave surface type algorithm which means that the warp yarns will not penetrate the fill yarns and vice-versa, but there may be a possibility of two warp or two fill yarns penetrating each other should they ever come into contact. For these single layer impact simulations, the first baseline serves to be the most robust. However as the size of this fabric model becomes larger, the first baseline becomes computationally very intensive compared to the other baselines in terms of both memory requirements and processing power. Also the third baseline remains intensive in terms of memory even though the processing power requirements compare to that of the second baseline. In such cases, the less intensive contact algorithm of the second baseline becomes preferable. These differences in computing requirements between the three baseline cases have been quantified and presented in Section 8. It is important to note here that with multiple fabric layers, an eroding type of contact (LSDYNA contact types 14 or 15) becomes necessary to prevent penetrations between the projectile and the fabric as well as between the various fabric layers, while the projectile is penetrating through. This type of contact algorithm is even more intensive than the first baseline used here. However by using different contact algorithms in different regions, much like our HEA model uses different finite elements and modeling resolutions in different regions of the fabric, the overall computational requirements can be reduced. One example would be to include a single surface eroding type contact algorithm for the region around the impact zone only, with a less intensive type of contact algorithm at far field regions from the impact zone where element erosion and penetration between two adjacent yarns is unlikely to occur. There is no clear choice as to which contact algorithm can be considered the best to use for all cases, since each contact algorithm has its own advantages as stated earlier. The choice will depend on the parameters and physics of the impact problem simulated and finally this is left to the experience of the FE analyst.

Fig. 12 compares the projectile velocity and fabric internal energy histories between the three baseline models for the $100 \mathrm{~m} / \mathrm{s}$ impact velocity case. The projectile is able to completely penetrate the fabric target with both impact velocity cases. The first instant of element erosion is slightly different in each baseline which triggers subsequent element failure at different times. Thus the projectile 

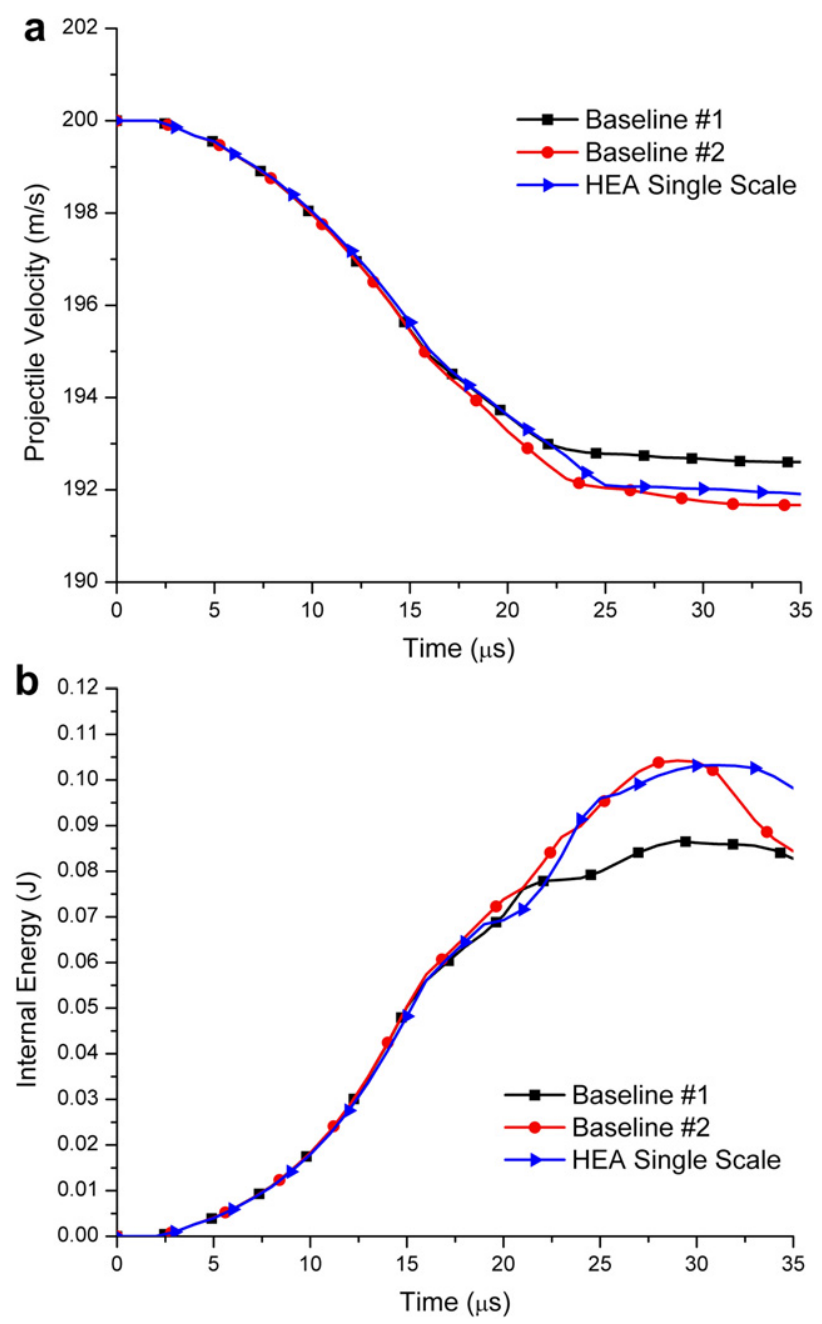

Fig. 17. Comparison between baseline and HEA models for impact velocity of $200 \mathrm{~m} / \mathrm{s}$ : (a) projectile velocity, (b) fabric internal energy. LS-DYNA contact algorithm for baseline \#1 (type 26), baseline \#2 (type a3).

velocity and fabric internal energy histories until the first instance of failure are exactly the same in all three baselines. However, even after complete penetration, the residual velocity of the projectile closely agrees between all baselines. The deformation of the fabric is pyramidal in nature, as seen in subsequent contour plots of fabric deformation profiles, and this agrees with experimental observations. The shape of the base of this pyramid is obtained by joining the front of the transverse displacement wave in each yarn by a line. When the outward propagating longitudinal strain wave reaches the four clamped boundaries, it reflects back towards the projectile. With each interaction of the longitudinal wave and the transverse displacement wave, the velocity of the transverse displacement wave is increased. The deformation pyramid does not reach the clamped boundaries before the projectile penetrates through the fabric in both impact velocity cases. While the projectile is penetrating through a hole in the fabric which is smaller than the projectile's diameter, the frictional energy dissipated by the projectile-yarn interaction rises to its maximum level. While the fabric is springing back after complete projectile penetration, the frictional energy dissipated by yarn-yarn sliding interactions rises to its maximum level.

This fabric model where the explicit yarn architecture is captured with solid elements is used as a baseline numerical model with which to compare the HEA models from subsequent sections. The HEA models will be compared to both the first and second baselines which respectively correspond to the most robust and computationally intensive (LS-DYNA type 26), and least computationally intensive contact algorithm (LS-DYNA type a3). The focus will be to reproduce the baseline fabric system response but at a lower computational expense.

\section{Hybrid element analysis applied to a fabric}

In multi scale modeling with the HEA method, different resolution levels modeled with different finite elements are incorporated into one single model. In this paper, the hybrid element analysis has first been applied to create single scale models, where the fabric is modeled using a yarn level architecture with both solid and shell elements. This combines the accuracy of yarn level resolution with solid elements, with the computational efficiency of shell elements. The region modeled with a yarn level architecture is referred to as the local region. Future work will deal with multi scale HEA models. In the future model, a homogenized membrane type region will be added to the model at far field locations and is referred to as the global region. Fig. 13 illustrates the various regions of a typical single scale HEA model. An explicit yarn architecture is used everywhere. For reasons cited earlier, solid elements have been used around the impact zone while shell elements have been used everywhere else. The HEA approach always ensures that the propagation of the longitudinal strain wave and transverse displacement wave across the solid-shell yarn interface is unaffected due to the impedance matching across the solid-shell interface. Fig. 14 is a close up at the impact zone, and also displays the mesh scheme. Due to symmetry only one quarter of the fabric has been modeled.

\section{Results and discussion}

A single scale HEA model is set up to compare results against the baseline numerical models described in Section 6. Consider the same fabric impact cases as the baselines, where the yarns have the same material properties. Yarn failure has been incorporated using an element erosion model with a maximum principal stress failure

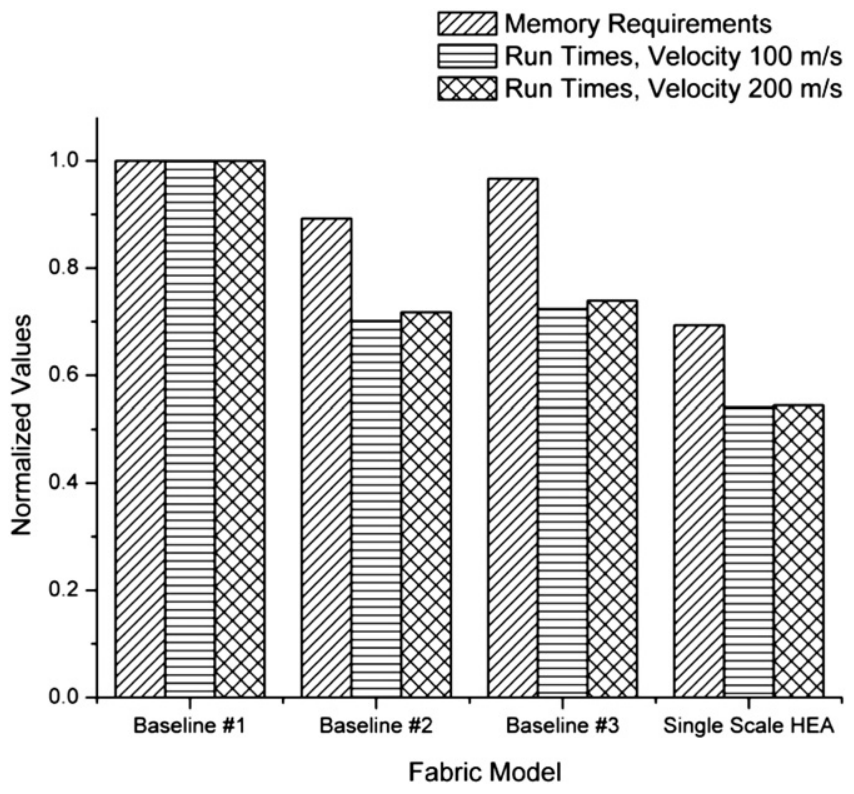

Fig. 18. Simulation memory requirements and run times. 


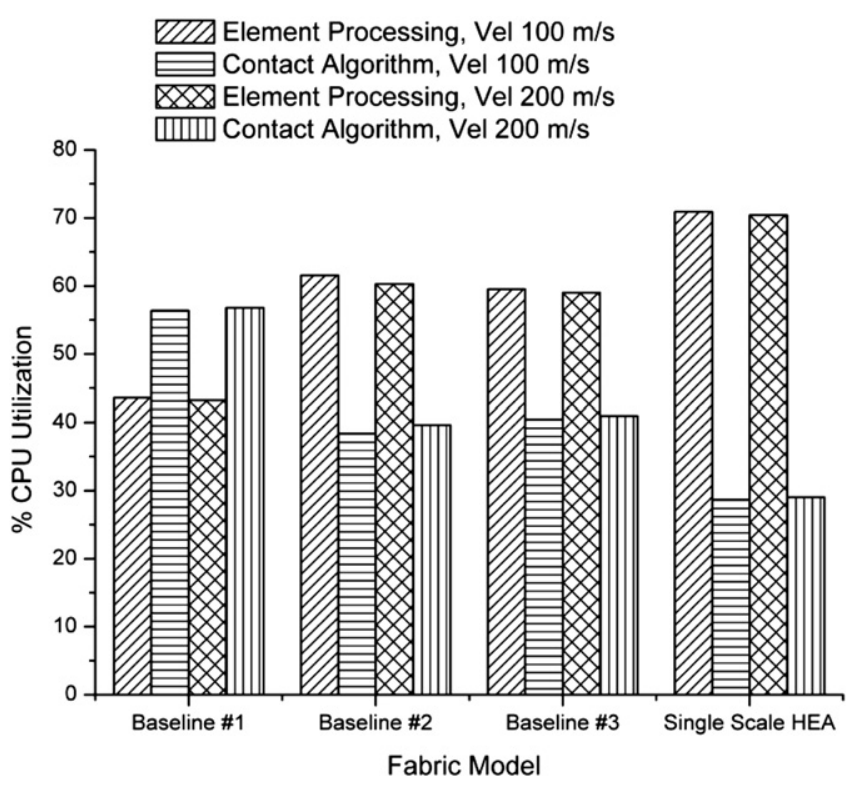

Fig. 19. Breakup of CPU utilization.

criterion of $3300 \mathrm{MPa}$. For the HEA model, the total dimensions of the central square patch with local yarn architecture modeled using solid elements are $9.5 \mathrm{~mm} \times 9.5 \mathrm{~mm}$ while the remaining fabric area consists of local yarn architecture modeled using shell elements. The ratio of the areas of the local solid element region to the local shell element region is 0.018 , and that of the shell element region to total fabric area is 0.982 . LS-DYNA contact type 26 is used for contact between the solid element yarns while contact type a3 is used for the shell element yarns. The interface between the solid and shell element yarns is created using the LS-DYNA keyword *CONSTRAINED_SHELL_TO_SOLID. The preprocessor DYNA-HEA [17] is used to automatically create the FE mesh and all interface definitions of the HEA model.

Fig. 15 compares the contours of vertical displacement between the first baseline and the HEA cases, as seen from the top view for the $100 \mathrm{~m} / \mathrm{s}$ impact velocity case. These contours are indicative of the propagation of the transverse displacement wave. The pyramidal deformation profile agrees with experimental observations. There is a good agreement between the deformation profiles. The transverse wave propagates slightly faster towards the two longer sides of the fabric because of the larger number of reflections of the longitudinal wave at the longer boundaries compared to the shorter boundaries. This implies that the number of interactions between the longitudinal wave and transverse displacement wave is greater along the direction of the shorter sides. The speed of the transverse displacement wave is increased with each interaction. The transverse displacement wave crosses the local-global interface by the time the projectile has penetrated through the fabric.

Fig. 16 compares the time history results of the baseline and HEA cases for the $100 \mathrm{~m} / \mathrm{s}$ impact velocity case. There is a very good agreement between the projectile velocity and fabric internal energy responses, especially prior to failure as seen in Fig. 16a and b. Fig. 16c compares the total sliding energy which is the energy dissipated when two surfaces slide past one another. This includes both projectile-yarn and yarn-yarn interactions. Since the fabric is gripped on all four sides, the sliding energy remains small until the projectile begins to penetrate through the fabric. After complete penetration, the fabric begins to elastically recover or spring back towards its initial shape which causes the sliding energy to rapidly increase. Fig. 16d compares the projectile to fabric contact force for all cases. This provides a measure of the resistance force that decelerates the projectile as well as the tension developed within the deformation pyramid. There is a very good agreement between the predictions.

Fig. 17 compares time history results of the baseline and HEA cases for the $200 \mathrm{~m} / \mathrm{s}$ impact velocity case. The first as well as subsequent instances of element failure vary between the baselines and the difference in the projectile velocity history after the onset of yarn failure becomes apparent starting at around $15 \mu \mathrm{s}$. This small difference is presumably due to the different contact algorithms used since all other parameters have been kept the same. There is a good agreement in the projectile velocity and fabric internal energy histories especially between the second baseline and the HEA cases.

Fig. 18 compares the computational requirements for both impact velocity cases. This consists of both the total run time as well as the memory requirements of all models. The simulations were run using LS-DYNA SMP version 971 on a 64-bit Dell Precision 690 workstation with four Intel Xeon $3.00 \mathrm{GHz}$ processors and $16 \mathrm{~GB}$ of available RAM. The single scale HEA model with fully local yarn architecture ran faster than all three baseline cases, and was approximately 2 times faster than the first baseline. These savings are not just reflected in the run times but also in the memory requirements of each model as seen in Fig. 18, where the HEA model required the least amount of memory. This is also an important consideration for reasons cited earlier.

This savings in computational expense while still reproducing the system response of the baseline simulations demonstrates the usefulness of the HEA approach. As the dimensions of the model and the simulation termination time increase, these savings become even larger. Fig. 19 displays the percentage utilization of the central processing unit ( $\mathrm{CPU}$ ) of the computer with respect to the run times reported in Fig. 18. In the first baseline case, we observe that the contact algorithm utilized more CPU resources than the processing of the finite elements. This was expected since this case corresponds to the most robust contact algorithm. We see this trend reversed in the other two baseline cases that used computationally less intensive contact algorithms while keeping the total number of finite elements and their formulation the same. Of all the cases, we observe that percentage CPU utilization of the element processing outweighed that of the contact algorithms the greatest in the single scale HEA case. Further even though this case used a fully local architecture, the relative CPU utilization by the contact algorithm was far less than that of the baselines, because the contact pairs defined were predominantly for shell yarn to shell yarn unlike the solid yarn to solid yarn pairs in the baseline cases.

\section{Conclusions}

The finite element modeling of the impact of flexible plain weave fabrics was discussed. A baseline fabric model comprising a yarn level architecture modeled with solid elements was presented. The use of different contact algorithms between the yarns in the finite element model was shown to have an effect on the fabric system response as well as the computational requirements. A novel approach, the hybrid element analysis, was introduced that incorporated using different finite element formulations currently at a single scale of modeling. Solid elements were used to model yarns at the impact zone while shell elements were used elsewhere. This choice of the appropriate finite element formulations to use in the various regions of the fabric model was based on the demonstrated inability of the shell elements to capture the correct stress response and yarn thickness changes in the impact zone directly underneath the projectile. The detrimental effects caused by a mismatch of impedance at the solid-shell interface were 
highlighted. The HEA approach was able to accurately reproduce the baseline simulation results but at a fraction of the computational expense, proving it to be an invaluable tool in the modeling and simulation of the impact of fabric systems. This research paper dealt with single scale modeling issues. Future work will deal with multi scale modeling issues using the HEA method, wherein an even greater savings in computational expense is envisioned with the inclusion of the homogenized or global region.

\section{Acknowledgements}

This work was supported by the US Army Research Laboratory at the Aberdeen Proving Ground, MD, USA through the Composite Materials Research (CMR) program at the University of Delaware, Center for Composite Materials.

\section{References}

[1] Tabiei A, Nilakantan G. Ballistic impact of dry woven fabric composites: a review. Applied Mechanics Reviews 2008;61(1):10801-13.

[2] Cheeseman BA, Bogetti TA. Ballistic impact into fabric and compliant composite laminates. Composite Structures 2003;61:161-73.

[3] Shahkarami A, Vaziri R. A continuum shell finite element model for impact simulation of woven fabrics. International Journal of Impact Engineering 2007;34:104-19.

[4] Lim CT, Shim VPW, Ng YH. Finite-element modeling of the ballistic impact of fabric armor. International Journal of Impact Engineering 2003;28:13-31.

[5] Ivanov I, Tabiei A. Loosely woven fabric model with viscoelastic crimped fibers for ballistic impact simulations. International Journal for Numerical Methods in Engineering 2004;61:1565-83.

[6] Gu B. Ballistic penetration of conically cylindrical steel projectile into plainwoven fabric target - a finite element simulation. Journal of Composite Materials 2004;38(22):2049-74.

[7] Duan Y, Keefe M, Bogetti TA, Powers B. Finite element modeling of transverse impact on a ballistic fabric. International Journal of Mechanical Sciences 2006;48:33-43.

[8] Zheng D, Binienda WK, Cheng J. Numerical modeling of friction effects on the ballistic impact response of single-ply tri-axial braided fabric. In: Proceedings of the ninth international LS-DYNA users conference, Dearborn, MI, USA, June 4-6, 2006.

[9] Wang Y, Miao Y, Cheeseman BA. Ballistic penetration simulation of textile fabrics. In: Proceedings of the Fiber Society 2006 fall annual meeting and technical conference, Knoxville, TN, USA, October 10-12, 2006.

[10] Wang Y, Sun X. Determining the geometry of textile preforms using finite element analysis. In: Proceedings of the American Society for Composites fifteenth annual technical conference, College Station, TX, USA, September 485-92, 2000. p. 485-92.

[11] Duan Y, Keefe M, Bogetti TA, Cheeseman BA. Modeling the role of friction during ballistic impact of a high-strength plain-weave fabric. Composite Structures 2005;68:331-7.

[12] Briscoe BJ, Motamedi F. Role of interfacial friction and lubrication in yarn and fabric mechanics. Textile Research Journal 1990;60(12):697-708.

[13] Barauskas R. Multi-scale modeling of textile structures in terminal ballistics. In: Proceedings of the sixth European LS-DYNA users conference, Gothenburg. Sweden, May 28-30, 2007.

[14] Barauskas R, Abraitiene A. Computational analysis of impact of a bullet against the multilayer fabrics in LS-DYNA. International Journal of Impact Engineering 2007; 34:1286-305.

[15] Rao MP, Keefe M, Powers BM, Bogetti TA. Multi-scale modeling of ballistic impact onto woven fabrics. In: Proceedings of the American Society for Composites twenty-second annual technical conference. Seattle, WA, USA, September 17-19, 2007. p. 51-69.

[16] Blankenhorn G, Schweizerhof K, Finckh H. Improved numerical investigations of a projectile impact on a textile structure. In: Proceedings of the fourth European LS-DYNA users conference, Ulm, Germany, May 22-23, 2003.

[17] Nilakantan G, Keefe M, Bogetti T, Gillespie Jr. JW. Novel multi-scale modeling of woven fabrics for use in impact studies. In: Proceedings of the tenth international LS-DYNA users conference, Dearborn, MI, USA, June 8-10, 2008.

[18] Gasser A, Boisse P, Hanklar A. Mechanical behavior of dry fabric reinforcements. 3D simulations versus biaxial tests. Computational Materials Science 2000;17:7-20.

[19] Duan Y, Keefe M, Bogetti TA, Cheeseman BA. Modeling friction effects on the ballistic impact behavior of a single-ply high-strength fabric. International Journal of Impact Engineering 2005;31:996-1012.

[20] Feynman RP, Leighton RB, Sands M. The Feynman lectures on physics, definitive edition, vol. 1. Addison-Wesley Publishing Company; 2006. ISBN 08053-9046-4.

[21] Tan VBC, Ching TW. Computational simulation of fabric armor subjected to ballistic impacts. International Journal of Impact Engineering 2006;32: 1737-51.

[22] Hallquist JO. LS-DYNA theory manual. Livermore Software Technology Corporation; March 2006. 